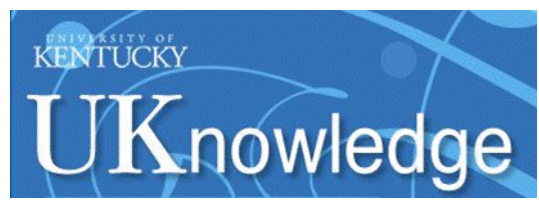

University of Kentucky

UKnowledge

$11-2010$

\title{
Structure and Feedback in 30 Doradus. I. Observations
}

\author{
E. W. Pellegrini \\ University of Michigan
}

J. A. Baldwin

Michigan State University

Gary J. Ferland

University of Kentucky, gary@uky.edu

Follow this and additional works at: https://uknowledge.uky.edu/physastron_facpub

Part of the Astrophysics and Astronomy Commons, and the Physics Commons

Right click to open a feedback form in a new tab to let us know how this document benefits you.

\section{Repository Citation}

Pellegrini, E. W.; Baldwin, J. A.; and Ferland, Gary J., "Structure and Feedback in 30 Doradus. I. Observations" (2010). Physics and Astronomy Faculty Publications. 141.

https://uknowledge.uky.edu/physastron_facpub/141

This Article is brought to you for free and open access by the Physics and Astronomy at UKnowledge. It has been accepted for inclusion in Physics and Astronomy Faculty Publications by an authorized administrator of UKnowledge. For more information, please contact UKnowledge@lsv.uky.edu. 


\section{Structure and Feedback in 30 Doradus. I. Observations}

Digital Object Identifier (DOI)

https://doi.org/10.1088/0067-0049/191/1/160

\section{Notes/Citation Information}

Published in The Astrophysical Journal Supplement Series, v. 191, no. 1, p. 160-178.

(c) 2010. The American Astronomical Society. All rights reserved.

The copyright holder has granted permission for posting the article here. 


\title{
STRUCTURE AND FEEDBACK IN 30 DORADUS. I. OBSERVATIONS
}

\author{
E. W. Pellegrini ${ }^{1,2,4}$, J. A. Baldwin ${ }^{2}$, and G. J. Ferland ${ }^{3}$ \\ ${ }^{1}$ Department of Astronomy, University of Michigan, 500 Church Street, Ann Arbor, MI 48109, USA; pelleger@ umich.edu \\ ${ }^{2}$ Physics and Astronomy Department, Michigan State University, 3270 Biomedical Physical Sciences Building, East Lansing, MI 48824, USA \\ ${ }^{3}$ Department of Physics and Astronomy, University of Kentucky, 177 Chemistry/Physics Building, Lexington, KY 40506, USA \\ Received 2010 August 4; accepted 2010 September 23; published 2010 October 27
}

\begin{abstract}
We have completed a new optical imaging and spectrophotometric survey of a $140 \times 80 \mathrm{pc}^{2}$ region of 30 Doradus centered on R136, covering key optical diagnostic emission lines including $\mathrm{H} \alpha, \mathrm{H} \beta, \mathrm{H} \gamma,[\mathrm{O}$ III] $\lambda \lambda 4363,4959$, 5007, [N II] $\lambda \lambda 6548,6584$, [S II] $\lambda \lambda 6717,6731$ [S III] $\lambda 6312$, and in some locations [S III] $\lambda 9069$. We present maps of fluxes and intensity ratios for these lines, and catalogs of isolated ionizing stars, elephant-trunk pillars, and edge-on ionization fronts. The final science-quality spectroscopic data products are available to the public. Our analysis of the new data finds that, while stellar winds and supernovae undoubtedly produce shocks and are responsible for shaping the nebula, there are no global spectral signatures to indicate that shocks are currently an important source of ionization. We conclude that the considerable region covered by our survey is well described by photoionization from the central cluster where the ionizing continuum is dominated by the most massive $\mathrm{O}$ stars. We show that if 30 Dor were viewed at a cosmological distance, its integrated light would be dominated by its extensive regions of lower surface brightness rather than by the bright, eye-catching arcs.
\end{abstract}

Key words: H II regions - ISM: individual objects (30 Doradus) - ISM: structure - surveys - techniques: spectroscopic

Online-only material: color figures, machine-readable table

\section{INTRODUCTION}

A significant share of our knowledge about star formation rates, chemical abundances, and abundance gradients in galaxies comes from studying emission lines from distant Giant Extragalactic H II Regions (GEHRs). But the observations of GEHRs are generally interpreted in the absence of a quantitative understanding of the structure responsible for the emission, or of its relationship to the processes of star formation. Massive stars heat, fragment, and compress the gas clouds from which they have recently formed. This "stellar feedback" is believed to drive the collapse of gravitationally bound clouds and trigger ongoing star formation (Zavagno et al. 2010; Oey et al. 2005; Elmegreen \& Lada 1977) and also shapes the structure of the gas which in turn affects the emission line spectrum. For these reasons, it is important to understand how such processes work.

Most GEHRs are distant, with poor spatial resolution. What is needed to better understand the mechanisms of stellar feedback is a nearby "Rosetta stone" in which we can resolve spatial details on the scale over which the feedback effects operate, for example, over the parsec-scale thickness of the ionized layer in an H II region. 30 Dor in the Large Magellanic Cloud, a nearby star-forming region that can be classified as a GEHR, is that key example. It is the largest star-forming region in the Local Group. The central cluster emits almost 500 times more ionizing photons than the Orion Nebula and 2-4 times more ionizing photons than other large Local Group star-forming regions such as NGC 3603 in our Galaxy or NGC 604 in M33. Because 30 Dor is only $48.5 \mathrm{kpc}$ away (Macri et al. 2006), it can be studied with high spatial resolution $\left(1^{\prime \prime}=0.25 \mathrm{pc}\right)$.

\footnotetext{
4 Visiting astronomer, Cerro Tololo Inter-American Observatory, National Optical Astronomy Observatory, which are operated by the Association of Universities for Research in Astronomy, under contract with the National Science Foundation.
}

The violent history of 30 Dor has led to the formation of vast cavities and shell-like structures around its central star cluster NGC 2070 (Meaburn 1984; Selman et al. 1999). The walls of the cavities have long been seen in optical ionization-front (IF) tracers like [S II]. The SAGE (Meixner et al. 2006) survey of the LMC included 30 Dor. SAGE traces the IFs in $8 \mu \mathrm{m}$ polycyclic aromatic hydrocarbon (PAH) emission with a resolution of 5 arcsec. Filling these cavities is a $10^{6} \mathrm{~K}$ gas visible in X-ray emission (Townsley et al. 2006). Many of these structures have $\mathrm{H} \alpha$ velocity profiles which show them to be expanding shells (Meaburn 1984). These shells have typical kinetic energies equal to $10^{51}$ erg with a total kinetic energy in the vicinity of NGC 2070 equal to $10^{52} \mathrm{erg}$ (Chu \& Kennicutt 1994). These types of kinematic arguments suggest supernovae ( $\mathrm{SNe})$ are a major component of the total energy budget. However, within reasonable estimates about the timescale for injecting energy from stellar winds into the interstellar medium it is equally likely that stellar winds are the agent shaping the nebula.

The remnants of the molecular gas cloud from which the stars have formed have been observed in optically thick CO emission (Poglitsch et al. 1995). This molecular cloud runs NE-SW behind NGC 2070. The brightest optical arcs, seen in Figure 1, appear to trace the surface of this molecular cloud, and are bright because of their high density. 30 Dor has been named the "Tarantula Nebula" because images showing emission lines are dominated by the interwoven pattern of these bright arcs. However, as we will show here, the majority of the line emission originates in the extended regions of low surface brightness.

This current paper describes our new optical-passband imaging and spectroscopic survey of 30 Dor. Our data set, which is publicly available, is designed to include key emission lines which quantitatively describe feedback processes. One of our goals is to survey the full region on the sky that would be lumped together in observations of a distant GEHR, for direct comparison to such objects. Luminous $\mathrm{H}$ II region complexes in distant 

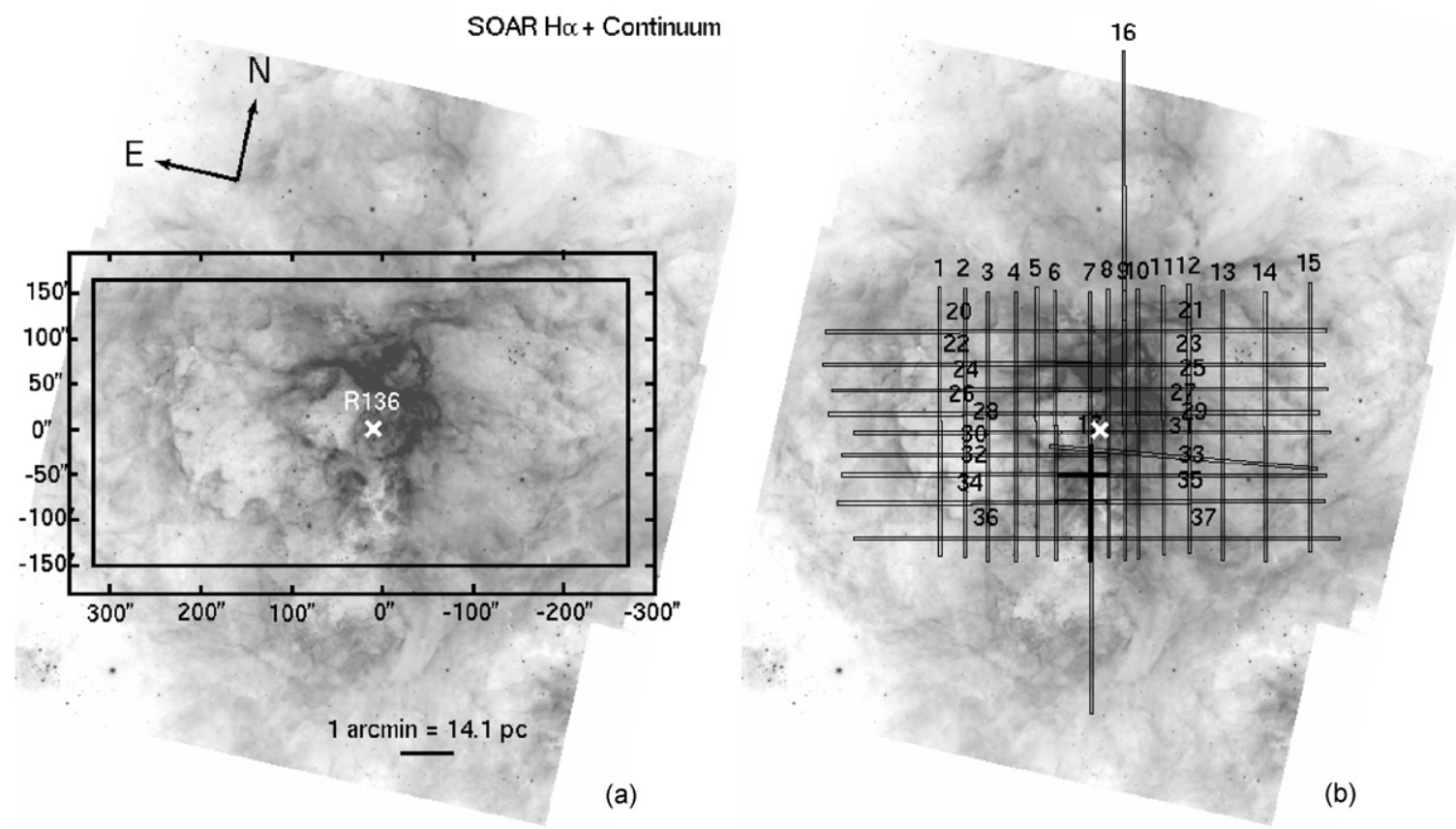

Figure 1. New narrowband $\mathrm{H} \alpha$ image of 30 Dor taken with the SOAR telescope. North is rotated $13^{\circ}$ clockwise from vertical. The center of R136 is marked as a white " $\times$ " symbol. (a) The outline of the region covered by our maps made from the Blanco spectra. (b) The individual slit positions of our Blanco spectroscopic data set.

galaxies typically are about $100 \mathrm{pc}$ in diameter (Oey et al. 2003; Hunt \& Hirashita 2009). The $140 \times 80 \mathrm{pc}^{2}$ area covered by our spectra is of similar size, and encompasses $50 \%$ of the $\mathrm{H} \alpha$ flux from the $10^{\prime}$ diameter region with $\mathrm{H} \alpha$ surface brightness appreciably above the general background level in this part of the LMC as measured from the Magellanic Cloud Emission Line Survey (MCELS; Smith \& MCELS Team 1998). The larger $11^{\prime} \times 12^{\prime}$ area of our subarcsecond resolution imaging survey encompasses $80 \%$ of the total $\mathrm{H} \alpha$ flux.

In 30 Dor, the numerous expanding shells are caused by mechanical energy input (Meaburn 1984; Chu \& Kennicutt 1994), and the ionizing energy source for a radius of at least $100 \mathrm{pc}$ is generally accepted to be photoionization by the central cluster NGC 2070 (Elliott et al. 1977; Tsamis et al. 2003). However, recent work based on Spitzer observations (Indebetouw et al. 2009, hereafter I09) has questioned this. In this paper, we use our observations to investigate the source of the photons that ionize the gas beyond the bright arcs.

The ionization and shaping need not be caused by the same mechanism. The application of our new data to studying feedback processes-the question of what has shaped the nebula - will be presented in a forthcoming companion paper (E. W. Pellegrini et al. 2010, in preparation, hereafter Paper II). There we will also discuss the consequences of the resulting structure on the escape of ionizing radiation. Finally, the second paper will evaluate the results from "strong line techniques" (Pagel et al. 1979; Kewley \& Dopita 2002; Denicoló et al. 2002) applied to our composite spectrum of 30 Dor, by comparing them to the chemical abundances and other nebular properties obtained by a point-by-point analysis of our spatially resolved data set.

\section{OBSERVATIONS}

\subsection{Existing Optical Passband Data Sets}

Most of the strong optical emission lines from star-forming regions trace ionized gas at electron temperatures $T_{e} \sim 10^{4} \mathrm{~K}$.
A few key lines from [O I], [O II], [N II], and [S II] form in the interface region at the edge of ionization-bounded $\mathrm{H}$ II regions. The elements S, O, N, Ar have transitions visible from the ground that are sensitive to both $T_{e}$ and the electron density $n_{e}$, and also to gas-phase abundances. Even though 30 Dor is a key example of a GEHR, the available measurements of it in the emission lines of these elements, using either direct imaging or especially spectroscopy, are surprisingly limited.

The best existing publicly available narrowband optical image data sets are the MCELS, and archival Hubble Space Telescope $(H S T)$ images. The MCELS survey covers the central $8 \times 8 \mathrm{deg}^{2}$ of the LMC including 30 Dor, in $\mathrm{H} \alpha+[\mathrm{N} \mathrm{II}]$, [O III] and [S II] (using many of the same emission line and continuum filters used in this present study). However, the data were taken with the $0.9 \mathrm{~m} \mathrm{Schmidt}$ telescope at Cerro Tololo Inter-American Observatory (CTIO) and have a spatial scale of 2.3 arcsec pixel $^{-1}$ with a resolution closer to 5 arcsec FWHM. This means that structures smaller than $1.2 \mathrm{pc}$ are unresolved, blurring fine details.

The HST archival data cover the central 4.5 by 3 arcmin of 30 Dor with at least 0.1 arcsec resolution (Scowen et al. 1998; Walborn et al. 2002) in $\mathrm{H} \alpha$, [O III] and [S II], revealing vastly more detail. However, the $H S T$ data are limited to the brighter central region around R136, the very compact group of stars at the very center of NGC 2070 . Since the brighter nebula accounts for only $25 \%$ of the total nebular emission in $\mathrm{H} \alpha$ the limited spatial coverage of HST misses the bulk of the emission that would be detected if 30 Dor were viewed from a much greater distance.

Turning to the available spectroscopy, Krabbe \& Copetti (2002) obtained a set of long-slit observations which covered $\mathrm{H} \beta$, [O $\mathrm{III}] \lambda 4363$, and $\left[\mathrm{O}_{\mathrm{III}}\right] \lambda 5007$ at 135 points along three slit positions. The sensitivity of these spectra is comparable to our Blanco survey described below. Mathis et al. (1985) had previously obtained spectra covering a similar wavelength range at four other slit positions. They presented line strengths measured 
Table 1

Summary of Imaging Observations Used in 30 Doradus Optical Mosaics

\begin{tabular}{|c|c|c|c|c|}
\hline Filter $(\AA)$ & $\begin{array}{c}\text { No. of Exp. } \times \text { Duration } \\
\text { at Each Position }\end{array}$ & No. Pos. & FWHM (arcsec) & Min/Max Exp Time (s) \\
\hline 6563 & $3 \times 150 \mathrm{~s}$ & 6 & 0.9 & $300 / 1480$ \\
\hline 6738 & $3 \times 450 \mathrm{~s}$ & 6 & 1.0 & $900 / 5700$ \\
\hline 6850 & $3 \times 450 \mathrm{~s}$ & 6 & $0.8-1.0$ & $900 / 5700$ \\
\hline 5019 & $3 \times 225 \mathrm{~s}$ & 5 & 1.0 & $450 / 2500$ \\
\hline 5130 & $3 \times 225 \mathrm{~s}$ & 5 & $1.0-1.1$ & $450 / 2500$ \\
\hline
\end{tabular}

for 22 extracted regions covering typically $30-60$ arcsec each along the slit.

Chu \& Kennicutt (1994) used the CTIO echelle spectrograph in a single-order mode to obtain a sparse grid of long-slit spectra that covered the $\mathrm{H} \alpha$ and [N II] $\lambda \lambda 6548,6584$ emission lines with high $\left(20 \mathrm{~km} \mathrm{~s}^{-1}\right)$ velocity resolution. They found that about half of the kinetic energy in 30 Dor is contained in shells in the central regions which are expanding with characteristic velocities $v \sim 20-200 \mathrm{~km} \mathrm{~s}^{-1}$, and that the kinetic energy contained in this expansion greatly exceeds the gravitational binding energy.

There has also been a limited amount of deep echelle spectroscopy covering a much wider wavelength range (Tsamis et al. 2003; Peimbert 2003). These spectra measured hundreds of emission lines that can be used for detailed chemical abundance analysis, and also cleanly resolve the density-sensitive [O II] $\lambda \lambda 3726,3729$ doublet and many other lines which are blended at lower spectral resolution. However, they require relatively long exposures and very short slit lengths, so have only very small areal coverage on 30 Dor. These existing measurements will be discussed below as needed.

\subsection{New Narrowband Images}

To the existing data sets we added a new set of narrowband images taken with the SOAR Optical Imager (SOI) on the $4.1 \mathrm{~m}$ SOAR Telescope. ${ }^{5}$ We used the $\mathrm{H} \alpha 6563 / 75$, [S II] 6738/ 50, [O III] 5019/15 emission-line filters and the 6850/100 and $5130 / 155$ continuum filters from the CTIO $3 \times 3$ and $4 \times 4$ in $^{2}$ filter sets, where the filter names refer to the approximate central wavelengths and FWHM bandpasses. A summary of the SOI observations is given in Table 1.

In each passband we took grids of $5 \times 5 \mathrm{arcmin}^{2} \mathrm{SOI}$ images, overlapping them to give a $12 \times 13 \mathrm{arcmin}^{2}$ field of view with a scale of 0.15 arcsec pixel $^{-1}$. The individual images were then combined to create final mosaic images in each passband. Due to the significant spatial overlap of the individual images, the total integration time in a given filter varies across the mosaic. Table 1 includes, for each filter, the resulting minimum and maximum integration times at any point in the mosaic. The data are seeing limited, with full width at half maximum intensity FWHM $=0.5-0.9$ arcsec, much better than the 5 arcsec FWHM in the MCELS survey, and are three times more sensitive to diffuse nebular emission than the existing $H S T$ data set.

As an example of the results, Figure 1(a) shows the mosaic image made with the $\mathrm{H} \alpha+[\mathrm{N}$ II] filter, prior to continuum subtraction. R136 is marked with a white $\times$. The 1 arcmin scale bar is equivalent to $14.1 \mathrm{pc}$ for an LMC distance of $48.5 \mathrm{kpc}$

\footnotetext{
5 The Southern Astrophysical Research (SOAR) Telescope is a joint project of Michigan State University, Ministério da Ciência e Tecnologia-Brazil, the University of North Carolina at Chapel Hill, and the National Optical Astronomy Observatory. Further information about SOAR and its instruments may be found at http://soartelescope.org.
}

(Macri et al. 2006). Figure 1(b) shows, on the same image, the slit positions used in the SOAR Telescope spectroscopic observations described below.

The two continuum filters were used to measure the emission from stars, together with the nebular continuum and starlight scattered off dust. The continuum image count rates were scaled and subtracted from the emission-line image to give a nebular count rate $R_{\text {line }}$ in each filter, according to

$$
R_{\text {line }}=R_{\text {narrow }}-R_{\text {cont }} \frac{W_{\text {narrow }}}{W_{\text {cont }}},
$$

where $R_{\text {narrow }}$ and $R_{\text {cont }}$ are equal to the atmospheric-extinction corrected count rate in the narrow-band and continuum filters, respectively, and the effective filter width $W_{i}$ in the $i$ th filter is

$$
W_{i}=\int T_{i}(\lambda) d \lambda
$$

where the transmission curve $T_{i}(\lambda)$ was measured by the SOAR staff.

Changes in stellar scattered light, seeing or sky brightness between the time the emission lines were measured and the continuum was measured were found to be a limiting factor in the quality of the continuum-subtracted images, so the observations with the continuum and emission filters were made sequentially one after the other before offsetting the telescope to each new point in the mosaic grid. This ensures that the relative flux between emission line and continuum images remains constant for each pointing.

\subsection{Spectrophotometry}

\subsubsection{The Blanco Telescope Spectral Grid}

In 2008 February, we obtained a grid of long-slit spectra of 30 Dor using the RC spectrograph on the $4 \mathrm{~m}$ Blanco telescope at CTIO. The spectrograph slit was 5 arcmin in length, and was positioned at a total of 37 different locations spanning the nebula (Figure 1(b)). Two sets of spectra were taken at orthogonal angles. The slit position angles P.A. $=13^{\circ}$ and P.A. $=103^{\circ}$ were chosen to maximize the number of key IFs that could be covered with the slit either crossing the IFs at an approximately perpendicular angle or running directly along them. The Blanco telescope uses an equatorial mount, which results in a constant position angle (P.A.) of the slit once the instrument has been rotated to the correct P.A. We minimized the uncertainty in the P.A. by rotating the slit only once each night, after all observations at the initial P.A. were completed. As a result, we have a high degree of confidence in our stated P.A.s, and therefore of the mapping onto the sky of individual points along the slit.

Table 2 lists for each slit position the identifying position number, the R.A. and decl. of the slit center in J2000 coordinates, 
Table 2

Summary of Blanco and SOAR Spectroscopic Observations of 30 Doradus

\begin{tabular}{|c|c|c|c|c|}
\hline Pos & R.A. (2000)(Slit Center) & Decl. (2000) & P.A. $(\operatorname{deg} \mathrm{E}$ of $\mathrm{N})$ & Exp. Time (s) \\
\hline \multicolumn{5}{|c|}{ Blanco } \\
\hline 1 & $05: 39: 16.53$ & $-69: 06: 37.04$ & 13 & 405 \\
\hline 2 & $05: 39: 11.25$ & $-69: 06: 31.18$ & 13 & 425 \\
\hline 3 & $05: 39: 06.29$ & $-69: 06: 29.68$ & 13 & 405 \\
\hline 4 & $05: 39: 00.44$ & $-69: 06: 22.80$ & 13 & 250 \\
\hline 5 & $05: 38: 56 . .29$ & $-69: 06: 11.60$ & 13 & 500 \\
\hline 6 & $05: 38: 52.21$ & $-69: 06: 11.81$ & 13 & 260 \\
\hline 7 & $05: 38: 45.00$ & $-69: 06: 03.65$ & 13 & 260 \\
\hline 8 & $05: 38: 41.38$ & $-69: 05: 55.66$ & 13 & 320 \\
\hline 9 & $05: 38: 37.98$ & $-69: 05: 53.44$ & 13 & 300 \\
\hline 10 & $05: 38: 35.17$ & $-69: 05: 48.71$ & 13 & 120 \\
\hline 11 & $05: 38: 30.08$ & $-69: 05: 38.24$ & 13 & 400 \\
\hline 12 & $05: 38: 24.82$ & $-69: 05: 29.57$ & 13 & 450 \\
\hline 13 & $05: 38: 17.42$ & $-69: 05: 28.52$ & 13 & 500 \\
\hline 14 & $05: 38: 08.61$ & $-69: 05: 18.33$ & 13 & 300 \\
\hline 15 & $05: 37: 59.70$ & $-69: 04: 56.19$ & 13 & 250 \\
\hline 16 & $05: 38: 49.47$ & $-69: 01: 28.18$ & 13 & 600 \\
\hline 17 & $05: 38: 37.48$ & $-69: 08: 52.47$ & 13 & 600 \\
\hline 20 & $05: 39: 16.50$ & $-69: 04: 51.76$ & 103 & 400 \\
\hline 21 & $05: 38: 28.34$ & $-69: 03: 50.99$ & 103 & 400 \\
\hline 22 & 05:39:15.65 & $-69: 05: 28.77$ & 103 & 405 \\
\hline 23 & $05: 38: 27.16$ & $-69: 04: 29.19$ & 103 & 308 \\
\hline 24 & $05: 39: 12.49$ & $-69: 05: 54.62$ & 103 & 500 \\
\hline 25 & $05: 38: 25.24$ & $-69: 04: 55.67$ & 103 & 355 \\
\hline 26 & $05: 39: 12.16$ & $-69: 06: 22.34$ & 103 & 500 \\
\hline 27 & $05: 38: 25.90$ & $-69: 05: 23.89$ & 103 & 460 \\
\hline 28 & $05: 39: 06.04$ & $-69: 06: 36.65$ & 103 & 600 \\
\hline 29 & $05: 38: 22.74$ & $-69: 05: 43.05$ & 103 & 520 \\
\hline 30 & $05: 39: 07.60$ & $-69: 07: 05.02$ & 103 & 700 \\
\hline 31 & $05: 38: 24.16$ & $-69: 06: 13.86$ & 98 & 450 \\
\hline 32 & 05:39:06.61 & $-69: 07: 26.38$ & 103 & 900 \\
\hline 33 & $05: 38: 21.35$ & $-69: 06: 31.58$ & 103 & 500 \\
\hline 34 & 05:39:06.01 & $-69: 07: 58.72$ & 103 & 400 \\
\hline 35 & $05: 38: 20.45$ & $-69: 07: 00.61$ & 103 & 300 \\
\hline 36 & 05:39:01.11 & $-69: 08: 34.64$ & 103 & 600 \\
\hline 37 & 05:38:15.70 & $-69: 07: 38.32$ & 103 & 450 \\
\hline \multicolumn{5}{|c|}{ SOAR } \\
\hline 40 & $05: 38: 46.430$ & $-69: 05: 35.06$ & 10 & 1000 \\
\hline 41 & 05:39:03.142 & $-69: 06: 40.45$ & 33.9 & 3000 \\
\hline 42 & 05:39:02.763 & $-69: 06: 45.82$ & 61 & 2000 \\
\hline 43 & $05: 39: 21.240$ & $-69: 06: 55.44$ & 61 & 300 \\
\hline 44 & 05:39:08.649 & $-69: 07: 02.80$ & 80 & 3000 \\
\hline
\end{tabular}

Notes. Columns 1-5 are position number corresponding to Figures 1 and 2, R.A. and declination of the slit center, P.A. and total integrated exposure time. Positions 1-37 are Blanco observations. 40-44 are SOAR observations.

the P.A., and the total exposure time. For simplicity, slit positions with P.A. $=13^{\circ}$ are numbered beginning at 1 at the easternmost position and increasing to 17 toward the west. These include the two most extreme north and south slit positions (position numbers 16 and 17, respectively). Numbering of slit positions with P.A. $=103^{\circ}$ then begins at 20 and increases toward the south, with even numbered slit positions centered to the east of R136 and odd numbered ones to the west. The only exception is position 31 which has a P.A. equal to $98^{\circ}$. Positions 40-44 were taken with the SOAR Telescope and are described in the following sub-section.

The Blanco Telescope observations were taken under photometric conditions with a slit width of 3.5 arcsec sampled at $0.5 \operatorname{arcsec}$ pixel $^{-1}$, using the $6001 \mathrm{~mm}^{-1}$ grating. This setup delivers a spectral resolution of $7.0 \AA$ (FWHM) with a spectral sampling of $1.06 \AA$ pixel $^{-1}$ as measured from the [O I] $\lambda 5577.33 \AA$ night sky line. Accurate offsetting was achieved by moving the autoguider probe by known amounts, and fiducial stars of known coordinates were placed in the slit at most of the slit positions in order to internally calibrate the position on the sky. An autoguider maintained the position to better than 1 arcsec.

The standard IRAF "LONGSLIT" package was used to generate bias and flat-field-corrected, flux-calibrated long-slit spectra, rectified to remove optical distortions and to convert to a linear wavelength scale. The wavelength solution had an rms uncertainty of $0.06 \AA$. After performing the distortion correction

\footnotetext{
6 IRAF is distributed by the National Optical Astronomy Observatory, which is operated by the Association of Universities for Research in Astronomy, Inc., under cooperative agreement with the National Science Foundation.
} 
using the wavelength calibration, any additional wavelength shifts due to flexure within the spectrograph were corrected by shifting each spectrum so that the [O I ] $\lambda 5577.33 \AA$ night sky line fell at the correct wavelength. The spectrophotometric standard stars LTT 2415, Hiltner 600, and CD32 from Hamuy et al. (1994) were observed on each night, to obtain an absolute flux calibration. A 7 arcsec slit was used for the flux calibrations to ensure that no light was lost in the wings of the stellar profile.

\subsubsection{Additional SOAR Telescope Spectroscopy}

Six additional locations in 30 Dor were observed with the Goodman High Throughput Spectrograph (Clemens et al. 2004) on the SOAR telescope, on 2009 February 5. The instrument was used with a single slit 3.9 arcmin long and 0.46 arcsec wide, with its 300 line $\mathrm{mm}^{-1}$ grating. The wavelength range was $3950 \AA$ to $9335 \AA$ with $1.32 \AA$ pixel $^{-1}$ sampling and a spectral resolution of $4.9 \AA \mathrm{FWHM}$ at $\mathrm{H} \alpha$. This setup easily resolved the [S II] doublet near $6720 \AA$ and the [O II] lines at 7320, $7330 \AA$.

Calibration of the data was performed with the spectroscopic packages in IRAF in a fashion similar to the method described above for the Blanco data, with a few notable exceptions. The spectrophotometric standard stars LTT 2415 and LTT 4816 were observed, without a slit, to obtain an absolute flux calibration. There is second-order contamination in the spectra beyond $7700 \AA$, with the sensitivity in second order below $3850 \AA$ A being about $36 \%$ of that in first order at the same wavelength. This is not a major problem for measurements of the nebular emission lines in the red (observed in first order), because at the positions of these lines the contaminating second-order light is mostly continuum emission. But the second-order contamination does strongly affect the flux calibration.

Second-order contamination in the red spectrum of the standard stars was accounted for by observing the standard stars through two different second-order blocking filters, GG385 and GG495, which cut off light blueward of 3850 and $4950 \AA$, respectively. The GG385 filter was used for the nebular observations and for the flux calibration for wavelengths lower than $7000 \AA$, while the GG495 filter was used only for the flux calibration beyond $7000 \AA$. To stitch together a flux calibration combined over the full wavelength range, a sensitivity response curve was generated separately for each filter, using the IRAF two-dimensional spectroscopic reduction package. A final response curve was made using the GG 385 curve below $7000 \AA$ and the GG495 curve at longer wavelengths.

Fringing with the current CCD is severe, reaching $32 \%$ at $9000 \AA$. To calibrate this, an internal quartz lamp was observed immediately after the series of nebular exposures at each slit position, before moving the telescope. In spite of this precaution, flexure within the instrument still caused significant, obvious offsets between the fringes in the nebular data and in the quartz calibration frame. We used the fringe pattern in the spectra of fiducial stars that fell in the slit as a guide for shifting and scaling in amplitude the quartz fringe frames before dividing out the fringe pattern. This procedure decreased the amplitude of the residual fringing to $10 \%$.

The SOAR spectra sample an area of very low $[\mathrm{S}$ II $] / \mathrm{H} \alpha$ intensity ratio, lying to the east of R136. Figure 2 shows the SOAR slit positions superimposed on a map of the $[\mathrm{S} \mathrm{II}] / \mathrm{H} \alpha$ intensity ratio made from our SOAR images. The orientation and spatial coverage of Figure 2 is the same as that of Figure 1. At the time of the observations, the SOAR Telescope lacked an atmospheric dispersion corrector, which is important with our broad wavelength coverage and narrow slit. As a result

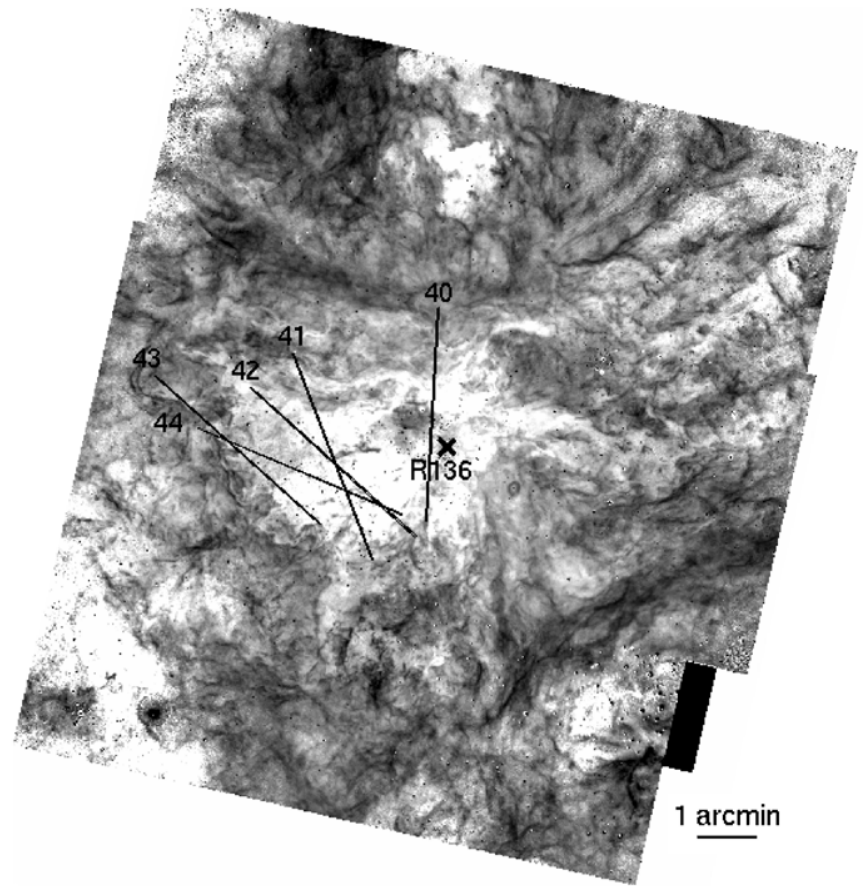

Figure 2. Ratio of [S II] $/ \mathrm{H} \alpha$ from our SOAR narrowband images. Darker shades indicate a higher ratio. The orientation is the same as Figure 1. For reference, the SOAR spectroscopic slit positions are plotted on top of the image.

our observations were restricted to nearly the parallactic angle, resulting in the pattern shown in Figure 2. The positions of the SOAR spectra are numbered beginning at 40 , to minimize confusion between the two spectroscopic data sets.

To remove night sky contamination we obtained a spectrum of the sky $2^{\circ}$ north and 2.7 east of R136. The sky spectrum was smoothed with a median filter spanning 15 arcsec along the slit, to decrease noise and remove stars along the slit. The intensity in the night sky lines was then scaled to match the object frames and subtracted. The redshift of the LMC helps to separate night sky forbidden lines from the same lines from 30 Dor. For example, in the case of the $[\mathrm{O}$ I] $\lambda 6300$ line, the nebular emission line is redshifted to $6306 \AA$. Figure 3 shows a typical spectrum before and after night sky subtraction, in the regions around $6300 \AA$ and $7330 \AA$.

The instrument setup used covers the low ionization [O I] $\lambda 6300$ and [O II] $\lambda \lambda 7320,7330$ lines as well as the highionization [S III] $\lambda 9068$ line and offers a clean unblended measurement of [S III] $\lambda 6312$ redshifted to $6318 \AA$. All of these lines except for [S III] $\lambda 9069$ are also within the wavelength range of the Blanco spectra but in that case are heavily blended with night sky lines and/or nebular emission and so were not measured. The region east of R136 was re-observed with SOAR because of the additional constraints which these lines provide when determining the ionization parameter $U$, the energy distribution of the ionizing radiation, and the $\mathrm{O} / \mathrm{H}$ and $\mathrm{S} / \mathrm{H}$ abundances.

\section{THE SPECTROSCOPIC "DATA CUBE"}

\subsection{Emission-line Flux Measurements}

One of our goals was to create a high-quality set of measured emission-line strengths that will be of general public use, so we will describe our measurement procedures in some detail. Table 3 lists the emission lines measured from the Blanco and SOAR spectra. Columns 1 through 3 indicate the observed and 


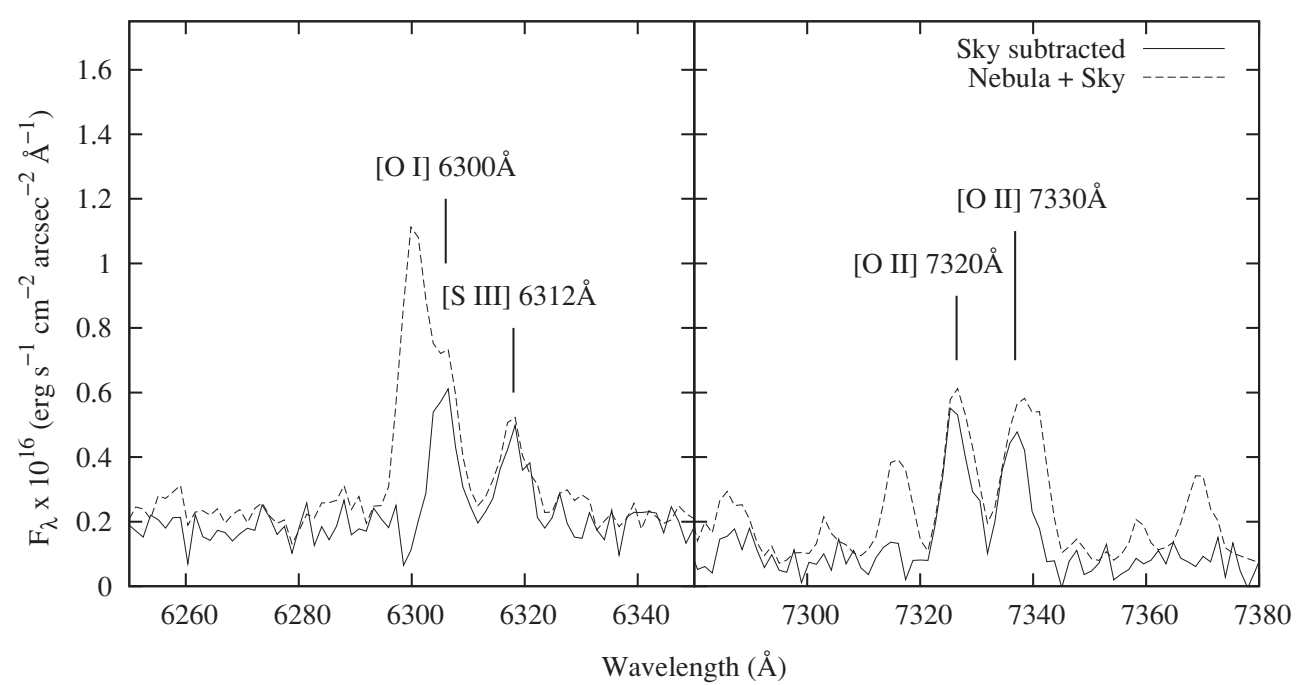

Figure 3. Sample SOAR spectrum near [O I] $\lambda 6300$ and [O II] $\lambda \lambda 7320,7330$. The solid and dashed lines respectively show the spectrum after and before sky subtraction.

Table 3

Line ID's and Wavelengths

\begin{tabular}{|c|c|c|c|c|}
\hline$\lambda_{\mathrm{Obs}}$ & $\lambda 0$ & Ion & $f_{\lambda}(R=3.1)$ & Data Set \\
\hline 4106 & 4101 & $\mathrm{HI}_{\mathrm{I}}$ & 1.43 & SOAR \\
\hline 4344 & 4340 & $\mathrm{HI}_{\mathrm{I}}$ & 1.35 & Blanco+SOAR \\
\hline 4366 & 4363 & [O III $]$ & 1.34 & Blanco+SOAR \\
\hline 4476 & 4471 & He I & 1.30 & Blanco+SOAR \\
\hline 4800 & 4800 & Continuum & 1.19 & Blanco+SOAR \\
\hline 4866 & $4861 *$ & HI & 1.16 & Blanco+SOAR \\
\hline 4926 & 4922 & $\mathrm{He}_{\mathrm{I}}$ & 1.14 & SOAR \\
\hline 4963 & 4959 & [O III] & 1.13 & Blanco+SOAR \\
\hline 5012 & $5007 *$ & [O III] & 1.12 & Blanco+SOAR \\
\hline 5625 & 5625 & Continuum & 0.97 & Blanco+SOAR \\
\hline 5880 & $5875^{*}$ & He I & 0.93 & Blanco+SOAR \\
\hline 6308 & 6300 & {$[\mathrm{OI}]$} & 0.86 & SOAR \\
\hline 6318 & 6312 & {$[\mathrm{~S}$ III] } & 0.86 & Blanco+SOAR \\
\hline 6364 & 6364 & NS [OI] & $\ldots$ & Blanco \\
\hline 6552 & 6548 & {$[\mathrm{~N} \mathrm{II}]$} & 0.82 & Blanco+SOAR \\
\hline 6570 & 6563 & $\mathrm{HI}$ & 0.82 & Blanco+SOAR \\
\hline 6590 & $6584^{*}$ & {$[\mathrm{~N}$ II $]$} & 0.81 & Blanco+SOAR \\
\hline 6684 & 6678 & He I & 0.80 & Blanco+SOAR \\
\hline 6721 & $6716^{*}$ & [S II $]$ & 0.79 & Blanco+SOAR \\
\hline 6738 & $6731 *$ & {$[\mathrm{~S} \mathrm{II}]$} & 0.79 & Blanco+SOAR \\
\hline 7072 & $7065^{*}$ & He I & 0.74 & Blanco+SOAR \\
\hline 7142 & $7135^{*}$ & [Ar III] & 0.73 & Blanco+SOAR \\
\hline 7288 & 7281 & He I & 0.71 & SOAR \\
\hline 7325 & 7320 & [O II] & 0.70 & SOAR \\
\hline 7337 & 7330 & [O II $]$ & 0.70 & SOAR \\
\hline 7758 & 7751 & [Ar III] & 0.63 & SOAR \\
\hline 9075 & 9069 & {$[\mathrm{~S} \mathrm{III]}]$} & 0.48 & SOAR \\
\hline
\end{tabular}

Notes. Measured strengths for each of these lines are listed in Table 4 and/or 5 at every extracted point in the nebula. Rest-frame wavelengths with an asterisk indicate the lines used to fit the Blanco data to models at each point in the nebula, as described in Paper II.

rest wavelengths and ionic species. The fourth column lists the extinction coefficient $f_{\lambda}$ used to deredden the observations, using a standard Galactic extinction curve with a ratio of total to selective extinction $R=3.1$ (Cardelli et al. 1989). The final column indicates which data sets include the emission line.

We extracted one-dimensional spectra binned over successive 2.5 arcsec increments along the slit in the two-dimensional spectra. The 2.5 arcsec width of the extraction window along the slit was chosen so that the in the fainter regions the line flux equaled the noise in the continuum. The program then automatically measured, in each extracted one-dimensional spectrum, the emission-line fluxes and their uncertainty. This produced a "data cube" of emission-line intensity measurements at 4238 points on the 30 Dor nebula.

We needed to use a summation approach to measure the flux in each emission-line profile because of the often complicated shape of the profiles. It is worth noting that most of this structure in the profiles is not due to velocity. It is due to spatial structure in the nebula that is smaller than the 3.5 arcsec slit width used for the Blanco spectra. To illustrate this, Figure 4(a) shows an enlarged portion of the SOAR [O III] image at the position of a ring-like structure, and Figure 4(b) shows the [O III] $\lambda 4959$ line in the two-dimensional image of the Blanco spectrum crossing this same location. The vertical lines on Figure 4(a) indicate the region over which the spectrum in Figure 4(b) was taken. The resulting emission-line structure in Figure 4(b) clearly reflects the spatial structure. This resulted in asymmetric profiles which were better measured by summing the total flux. Figure 4(c) shows the profile extracted over the area along the slit marked in the other panels, with a best-fitting Gaussian shape superimposed to demonstrate that the line shape is not well represented by a simple Gaussian.

The wavelength windows used in the automated linemeasuring procedure were manually set in advance, using the brightest regions of the nebula. Two examples of wavelength windows are presented in Figure 5. In the case of an isolated line like He I $\lambda 6678$, two "wing" boundaries are chosen to include all of the line flux shown by the vertical dashed lines. The continuum regions (indicated by horizontal dashed lines) are separately defined on either side of the emission line, avoiding other emission lines. In the case of mildly blended lines, like the [S II] $\lambda \lambda 6716,6731$ doublet, the wings are defined to include the total flux from both lines. A search is then made for the minimum value in the area between the two line peaks (over the range indicated by the width of the solid horizontal bar in the example shown in Figure 5), to define the point of separation between the extraction windows of the two individual lines. This does not perfectly deblend the two lines, but since the ratio of $[\mathrm{S} \mathrm{II}] \lambda 6716 / \lambda 6731$ is always of order unity the error is negligible.

This procedure for measuring the line strengths works well even in the cases where stars are present in the extraction 

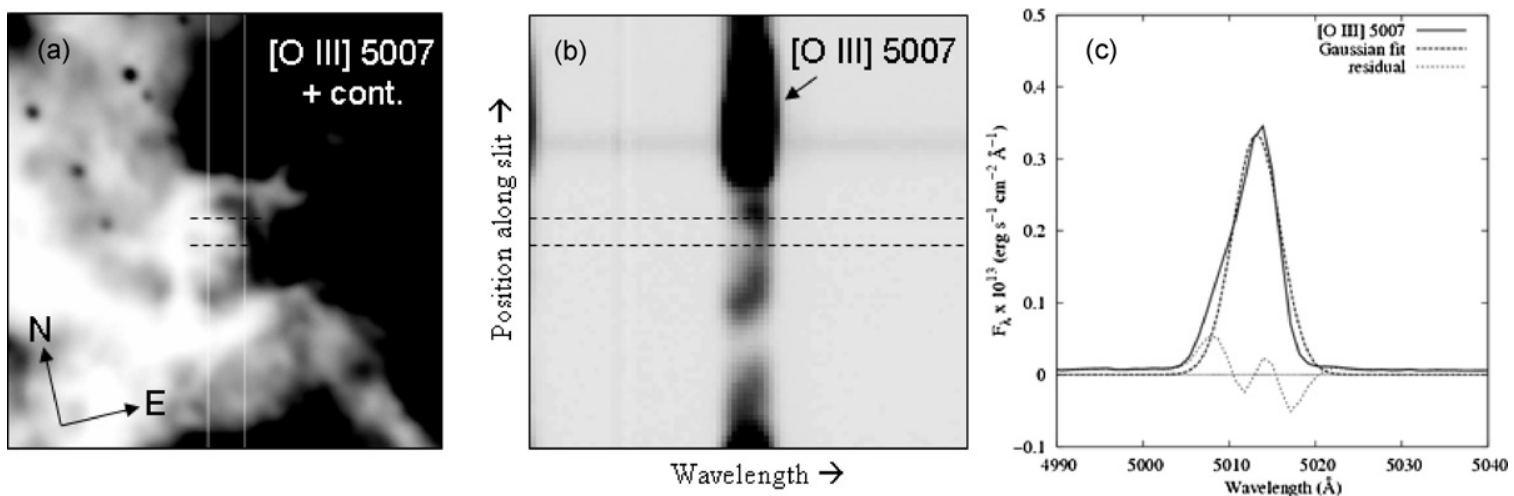

Figure 4. Demonstration that the structure in the line profiles is dominated by spatial structure in the nebula rather than by velocity structure. Panel (a) is a twodimensional image of the sky in [O III] emission. Panel (b) shows, on the same spatial scale as panel (a), the two-dimensional Blanco spectrum measured with a 3.5 arcsec wide slit indicated by the vertical solid lines shown in panel (a). The dispersion is in the horizontal direction. Panel (c) shows the [O III] $\lambda 5007$ profile in an extracted two-dimensional spectrum summed over the portion of the slit between the dotted lines shown in panels (a) and (b). In panel (c), the top dashed line is the best Gaussian fit and the bottom dashed line is the residual from that fit. The non-Gaussian shape of the [O III] line in panel (c) clearly is due to the spatial structure seen in panel (a).

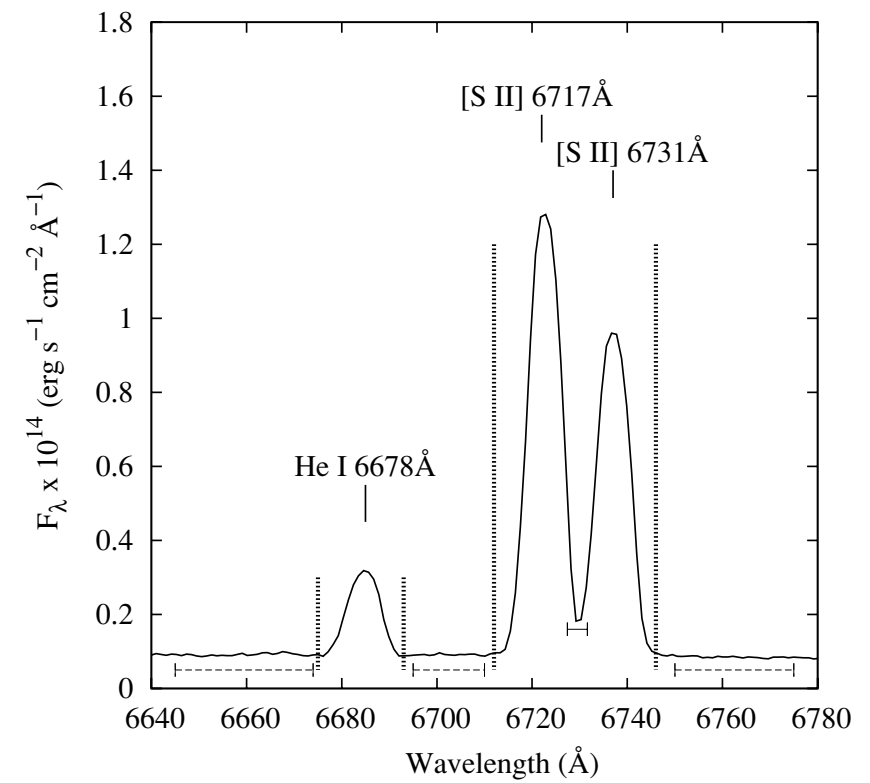

Figure 5. Two examples of extraction windows used to measure line flux. The dashed horizontal bars show the ranges over which the continuum was fitted. The vertical lines show the ranges over which the line fluxes were summed. He I $\lambda 6678$ is an example of an isolated emission line, while the [S II] doublet is slightly blended. The solid horizontal bar between the two [S II] peaks shows the range over which a search was performed for the minimum value, to define the boundary point between the two individual $\left[\mathrm{S} \mathrm{II}_{\mathrm{II}}\right.$ lines, as described in the text.

region, as long as the star is not extremely bright or the nebula extremely faint. Possible problem cases were identified using the continuum bins, and were checked manually and for a few cases all emission lines at that position were excluded. In the case of bright stars with strong absorption features the surface brightness of $\mathrm{H} \beta$ will be underestimated. Placement of bright star clusters in the slit was intentionally avoided and the number of regions affected by stellar absorption is small.

\subsection{Noise Estimates}

We estimated the uncertainties in the measured line strengths by assuming Poisson statistics in the original photon count rate and then carrying parallel noise images all the way through the same reduction process as the data images. At the beginning of the data reduction the measured counts were converted to photons using the known detector gain. An initial noise image was then calculated using

$$
\sigma=\left(N_{\text {photons }}+\text { gain } \times \text { ReadNoise }_{\text {electrons }}^{2}\right)^{1 / 2} .
$$

All multiplicative calibrations such as flat fields, illumination corrections, and the flux calibration were applied to the noise image by multiplying the $\sigma$ values in the noise image.

In the step where the spectra were linearized and distortion was corrected, there was a rebinning of the data. In this case, $\sigma^{2}$ (the variance of the noise) was rebinned using the same transformation. The resulting $\sigma$ in each pixel is thus summed in quadrature. We manually inspected a random set of extracted spectra and verified that these estimated errors do in fact reasonably measure the observed pixel-to-pixel scatter in the continuum points. An exception to this is [O III] $\lambda 4363$, where uncertainties in the continuum level and blending with the wings of H I $\lambda 4340$ cause special problems, as is discussed below. The $\sigma$ values for each pixel were then used to compute error bars for the measured line fluxes and ratios.

\subsection{Detector Saturation}

The large range in the surface brightness of the nebula meant that the 5 arcmin long slit included both bright and faint regions. In order to get a sufficiently high signal-to-noise ratio in the faint regions, long exposures were used and the [O III] $\lambda 5007$ and/or $\mathrm{H} \alpha$ lines were often saturated at various places along the slit. When necessary, additional short exposures were made to correct for saturation. All repeated observations of a single position, long and short, were gray shifted to the highest value according to the total flux of the [O III] $\lambda 4959$ line summed over the full 300 arcsec length of the slit. The $\lambda 4959$ line was used because it is a moderately strong line but is never saturated. Summing along the slit minimizes any effects of variable seeing or telescope drift.

As a final check to the data, line ratios of extracted regions were compared wherever two slits intersected. While the extracted regions at these points overlap, they do not sample completely identical parts of the nebula. The normalized histogram shown in Figure 6 demonstrates the repeatability of the $[\mathrm{O}$ III $] \lambda 5007 / \mathrm{H} \beta$ and $([\mathrm{S}$ II $] \lambda 6716+\lambda 6731) / \mathrm{H} \alpha$ ratios measured for these overlapping observations. The histogram shows that 


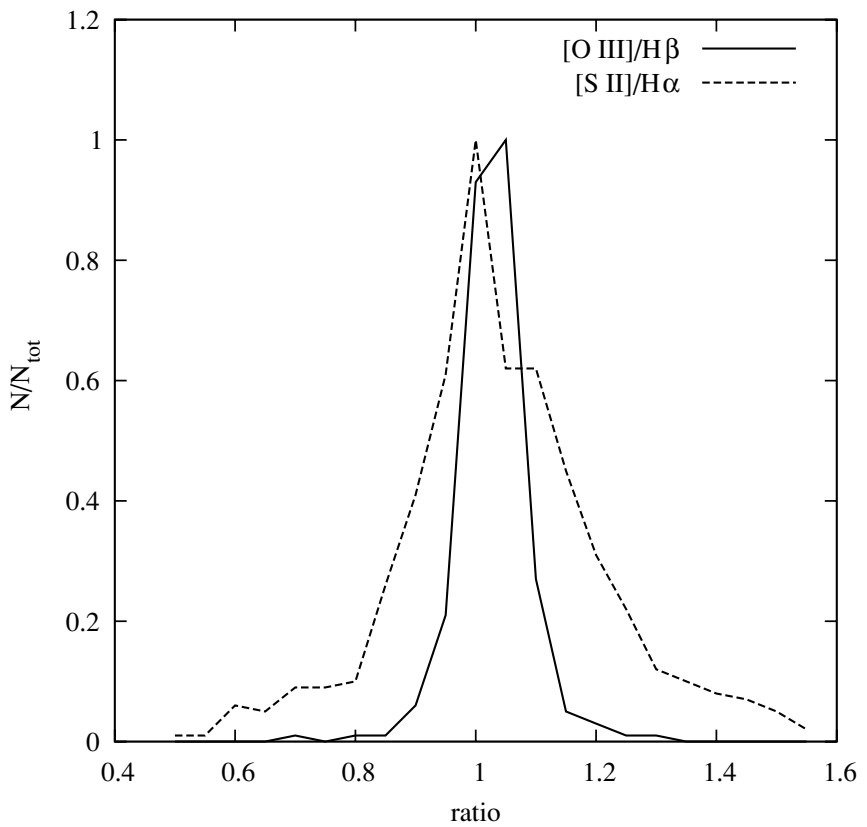

Figure 6. Repeatability of results at overlapping points along different Blanco slit positions. The curves are histograms of the distributions of ratios of intensity ratios; for example, the $[\mathrm{O} \mathrm{III}] / \mathrm{H} \beta$ measured from one slit position is divided by the $[\mathrm{O} \mathrm{III}] / \mathrm{H} \beta$ measure at the same position on the sky but from a different slit position.

the data do repeat to within the typical range of differences due to the mismatch between the areas sampled on the sky by the two slits, with especially good repeatability in the $[\mathrm{O} \mathrm{III}] / \mathrm{H} \beta$ ratio.

\subsection{Reddening Correction}

The reddening was determined separately for each extracted one-dimensional spectrum, using the observed $\mathrm{H} \alpha / \mathrm{H} \beta$ intensity ratio and assuming an intrinsic ratio of 2.87 appropriate for Case $\mathrm{B}$ and a gas temperature $10^{4} \mathrm{~K}$ (Osterbrock \& Ferland 2006) and $R=3.1$. This is adequate even though 30 Dor is in the LMC because (1) although the LMC extinction curve departs considerably from the Galactic curve in the ultraviolet, the two are very similar in the optical passband, and (2) some considerable part of the reddening is in any case due to foreground material within our own Galaxy. We then applied the reddening to each measured line from the same extracted one-dimensional spectrum, using the extinction curve values $f_{\lambda}$ from Table 3. A check on the validity of this procedure is that the dereddened $\mathrm{H} \gamma / \mathrm{H} \beta$ ratios agree with the predicted Case B values to within an average of $3 \%$, with a $1 \sigma$ scatter of $4 \%$ which is consistent with the observational errors.

\subsection{Electron Density and Temperature}

At each position along each spectrograph slit we determined the electron density $n_{e}$ from the dereddened [S II] $\lambda 6716 / \lambda 6731$ line ratio. The IRAF/STSDAS task nebular was used to derive the electron densities using a 5 level sulfur atom assuming a gas temperature of $10^{4} \mathrm{~K}$. The errors in the density measurements were calculated using the $1 \sigma$ uncertainty in the line ratio. The difference between the densities at these extremes and at the nominal value is the reported density uncertainties. If either the nominal or $1 \sigma[\mathrm{S}$ II] line ratio was greater than 1.41 the density was only constrained to be $n_{e} \leqslant 10 \mathrm{~cm}^{-3}$ (the low-density limit). Using the published contour plots of $\log \left(n_{e}\right)$ derived from
[S III] in I09 we compared the electron densities measured from [S II] and [S III]. We find they agree to $0.1-0.2$ in $\log \left(n_{e}\right)$, noting that the increments in the density contours of I09 are 0.1 in $\log \left(n_{e}\right)$.

The electron kinetic temperature $T_{e}$ was also measured at each position, from the dereddened [O III] $(\lambda 5007+\lambda 4959) /$ $\lambda 4363$ ratio again using the IRAF/STSDAS nebular task. The density used in the calculation is from the [S II] lines. Errors were computed in the same way as for the density values.

\subsection{A Publicly Available Data Set}

The Blanco instrument setup was chosen to cover the strong optical nebular emission lines from 4100 to $7400 \AA$ including $\mathrm{H} \beta, \mathrm{H} \alpha$, and the $[\mathrm{S} \mathrm{II}]$ doublet at $\lambda \lambda 6716,6731$. The Blanco observations were made in the form of a grid with fairly regular spacing which allow the data to be turned into a "data cube."

Our Blanco slit positions were chosen to produce a relatively unbiased sample of the diffuse emission as well as to include discrete structures of interest. Post-observing verification of the location of each slit position on the sky was done using narrowband emission line and continuum images taken at the SOAR telescope by (1) comparing the extracted surface brightness profile of $\mathrm{H} \alpha$ along the slit to the $\mathrm{H} \alpha$ images, (2) using positions, measured from our narrowband continuum images, of stars purposely placed in the slit as references, and (3) where necessary using two-dimensional nebular features visible in both the spectra and images such as in the example shown in Figure 4.

Table 4 is a sample of the final data product from the Blanco spectra. It lists the dereddened line strengths and several additional parameters for each extraction window along each slit position, and their uncertainties. The entire table, which is available in electronic format in the online edition of the journal, includes measurements at 4238 positions with one row per position. Column 1 lists the slit position number (as used in Figure 1(b) and Table 2). Columns 2-4 contain the R.A. and decl. offsets in arcsec from R136 and the central pixel row of the extraction window along the slit. Then columns 5-10 list the electron density and its $1 \sigma$ uncertainty limits, followed by the electron temperature with its $1 \sigma$ uncertainties. Column 11 is the reddening $A_{V}$ deduced from the $\mathrm{H} \alpha / \mathrm{H} \beta$ ratio. These are followed in Columns 12 and 13 by the dereddened $\mathrm{H} \beta$ surface brightness and its $1 \sigma$ uncertainty. The remaining 44 columns list, in pairs, the dereddened surface brightness of each measured emission line relative to $\mathrm{H} \beta$ and the corresponding uncertainty.

The last three rows of Table 4, collectively labeled position 38 , list the average properties of 30 Doradus derived from the entire data set. Position 38 row 1 is the average of the dereddened fluxes, and the physical properties derived from those. Row 2 is the average of the observed values. Row 2 was then dereddened using the average $\mathrm{H} \beta$ and $\mathrm{H} \alpha$ fluxes to produce row 3. Rows 2 and 3 represent the global spectroscopic properties that would be measured for 30 Dor if it were spatially unresolved.

Table 5 is the similar data product from the SOAR spectra, computed in exactly the same way as for Table 4 . The SOAR spectra are presented in a separate table because there are a different number of columns due to the additional emission lines which were measured, including [S III] $\lambda$ 9069. The ratio [S III] $\lambda 9069 / \lambda 6312$ is sensitive to the gas temperature like $[\mathrm{O}$ III $] \lambda 5007 / \lambda 4363$. The temperatures measured from the [S III] lines are included in Table 5. The complete table is available from the author upon request. 
Table 4

Sample of Blanco Spectroscopy Data Cube

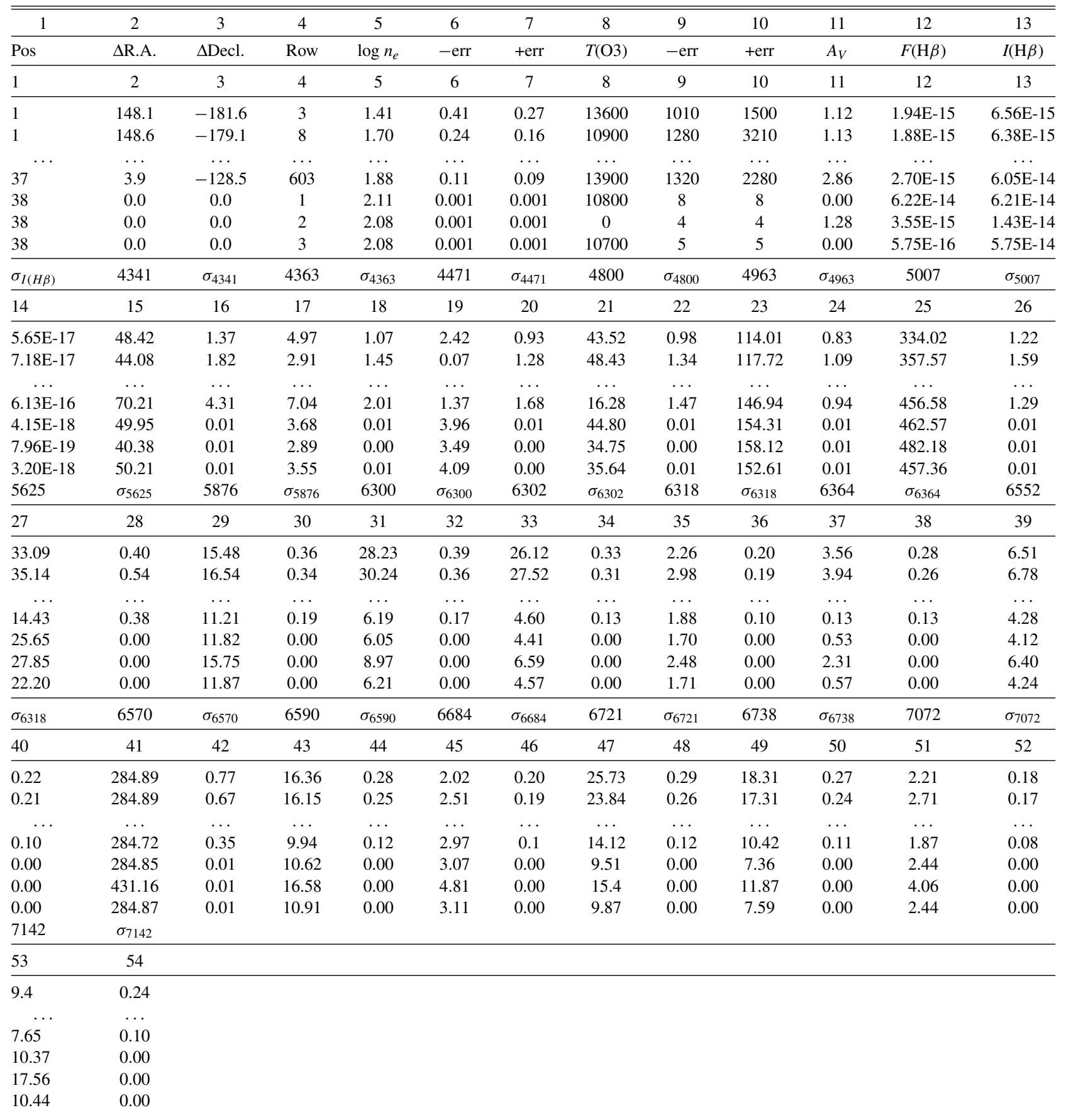

Notes. The units of electron density and temperature are $\mathrm{cm}^{-3}$ and $\mathrm{K}$, respectively. The dereddened $\mathrm{H} \beta$ surface brightness is reported in units of $\mathrm{erg} \mathrm{s}^{-1} \mathrm{~cm}^{-2}$ arcsec ${ }^{-2}$. The strengths of the other emission lines are given in units of $100 \times \mathrm{S}($ line $) / \mathrm{S}(\mathrm{H} \beta)$. The entries for position number 38 are average values for the whole data set, as described in the text.

(This table is available in its entirety in a machine-readable form in the online journal. A portion is shown here for guidance regarding its form and content.)

In addition to the tabulated data, the fully reduced Blanco two-dimensional spectra, noise, and calibration frames are publicly available. They are posted on a permanent Web site at the Michigan State University Department of Physics \& Astronomy.

\footnotetext{
7 http://www.pa.msu.edu/astro/thesis/pellegrini/30dor/
}

\section{OBSERVATIONAL RESULTS}

\subsection{Overview}

Figures 7-12 are maps of selected emission lines, nebular diagnostics, and physical conditions derived from those emission lines, interpolated in the spatial plane of the Blanco data cube. The two extreme slit positions, 16 and 17, are excluded from 
Table 5

Sample of SOAR Data Spectroscopy Data Set

\begin{tabular}{|c|c|c|c|c|c|c|c|c|c|c|c|}
\hline 1 & 2 & 3 & 4 & 5 & 6 & 7 & 8 & 9 & 10 & 11 & 12 \\
\hline Pos & $\Delta$ R.A. & $\Delta$ decl. & Row & $\log n_{e}$ & -err & +err & $T(\mathrm{O} 3)$ & -err & +err & $T(\mathrm{~S} 3)$ & -err \\
\hline 1 & 2 & 3 & 4 & 5 & 6 & 7 & 8 & 9 & 10 & 11 & 12 \\
\hline 40 & 4.65 & -72.58 & 260 & 2.34 & 0.06 & 0.05 & 13100 & 582 & 728 & 11100 & 603 \\
\hline 40 & 5.03 & -70.42 & 275 & 2.43 & 0.08 & 0.08 & 14100 & 1120 & 1720 & 9250 & 1000 \\
\hline 40 & 5.41 & -68.26 & 290 & 2.67 & 0.06 & 0.06 & 11500 & 1050 & 1890 & 10900 & 917 \\
\hline$\ldots$ & $\ldots$ & $\ldots$ & $\ldots$ & $\ldots$ & $\ldots$ & $\ldots$ & $\ldots$ & $\ldots$ & $\ldots$ & $\ldots$ & $\ldots$ \\
\hline +err & $A_{V}$ & $F(\mathrm{H} \beta)$ & $I(\mathrm{H} \beta)$ & $\sigma_{I(H \beta)}$ & 4106 & $\sigma_{4106}$ & 4344 & $\sigma_{4344}$ & 4368 & $\sigma_{4368}$ & 4474 \\
\hline 13 & 14 & 15 & 16 & 17 & 18 & 19 & 20 & 21 & 22 & 23 & 24 \\
\hline 775 & 1.49 & $3.78 \mathrm{E}-14$ & $1.10 \mathrm{E}-13$ & $5.80 \mathrm{E}-16$ & 24.646 & 1.631 & 53.838 & 1.009 & 6.897 & 0.851 & 2.777 \\
\hline 1960 & 1.89 & $1.87 \mathrm{E}-14$ & 8.13E-14 & 7.49E-16 & 32.489 & 3.662 & 53.265 & 2.086 & 8.330 & 1.869 & 3.477 \\
\hline 1400 & 1.88 & $2.08 \mathrm{E}-14$ & $9.07 \mathrm{E}-14$ & $7.60 \mathrm{E}-16$ & 34.825 & 3.258 & 53.366 & 1.866 & 4.986 & 1.650 & 2.839 \\
\hline$\ldots$ & $\ldots$ & $\ldots$ & $\ldots$ & $\ldots$ & $\ldots$ & $\ldots$ & $\ldots$ & $\ldots$ & $\ldots$ & $\ldots$ & $\ldots$ \\
\hline$\sigma_{4474}$ & 4800 & $\sigma_{4800}$ & 4926 & $\sigma_{4926}$ & 4963 & $\sigma_{4963}$ & 5012 & $\sigma_{5012}$ & 5522 & $\sigma_{5522}$ & 5542 \\
\hline 25 & 26 & 27 & 28 & 29 & 30 & 31 & 32 & 33 & 34 & 35 & 36 \\
\hline 0.676 & 24.593 & 0.622 & 0.793 & 0.267 & 166.054 & 0.547 & 502.391 & 0.830 & 3.189 & 0.105 & 2.360 \\
\hline 1.456 & 47.975 & 1.293 & 3.382 & 0.548 & 169.743 & 0.902 & 510.836 & 1.287 & 5.577 & 0.202 & 3.876 \\
\hline 1.289 & 45.377 & 1.151 & 0.228 & 0.486 & 168.095 & 0.827 & 512.208 & 1.196 & 5.368 & 0.181 & 4.101 \\
\hline $\begin{array}{c}\cdots \\
\sigma_{5542} \\
\end{array}$ & $\begin{array}{c}\ldots \\
5625\end{array}$ & $\begin{array}{c}\ldots \\
\sigma_{5625} \\
\end{array}$ & $\begin{array}{c}\ldots \\
5881\end{array}$ & $\begin{array}{c}\ldots \\
\sigma_{5881} \\
\end{array}$ & 6306 & $\begin{array}{c}\cdots \\
\sigma_{6306} \\
\end{array}$ & 6318 & $\begin{array}{c}\cdots \\
\sigma_{6318} \\
\end{array}$ & 6552 & $\begin{array}{c}\ldots \\
\sigma_{6552} \\
\end{array}$ & $\begin{array}{c}\ldots \\
6570\end{array}$ \\
\hline 37 & 38 & 39 & 40 & 41 & 42 & 43 & 44 & 45 & 46 & 47 & 48 \\
\hline 0.096 & 10.438 & 0.196 & 12.476 & 0.133 & 0.948 & 0.064 & 1.501 & 0.067 & 2.560 & 0.064 & 285.000 \\
\hline 0.185 & 16.704 & 0.372 & 12.888 & 0.236 & 0.415 & 0.116 & 0.966 & 0.119 & 2.360 & 0.111 & 285.000 \\
\hline 0.166 & 17.714 & 0.334 & 13.428 & 0.215 & 0.703 & 0.105 & 1.457 & 0.109 & 2.257 & 0.100 & 285.000 \\
\hline$\ldots$ & $\ldots$ & $\ldots$ & $\ldots$ & $\ldots$ & $\ldots$ & $\ldots$ & $\ldots$ & $\ldots$ & $\ldots$ & $\ldots$ & $\ldots$ \\
\hline$\sigma_{6570}$ & 6590 & $\sigma_{6590}$ & 6684 & $\sigma_{6684}$ & 6722 & $\sigma_{6722}$ & 6736 & $\sigma_{6736}$ & 7072 & $\sigma_{7072}$ & 7144 \\
\hline 49 & 50 & 51 & 52 & 53 & 54 & 55 & 56 & 57 & 58 & 59 & 60 \\
\hline 0.284 & 7.993 & 0.081 & 3.462 & 0.067 & 6.128 & 0.067 & 5.027 & 0.065 & 2.284 & 0.052 & 9.834 \\
\hline 0.394 & 7.911 & 0.134 & 3.414 & 0.115 & 5.937 & 0.109 & 5.010 & 0.109 & 2.436 & 0.088 & 9.705 \\
\hline 0.369 & 6.979 & 0.120 & 3.163 & 0.104 & 5.005 & 0.097 & 4.697 & 0.098 & 2.420 & 0.080 & 9.577 \\
\hline$\ldots$ & $\ldots$ & $\ldots$ & $\ldots$ & $\ldots$ & $\ldots$ & $\ldots$ & $\ldots$ & $\ldots$ & $\ldots$ & $\ldots$ & $\ldots$ \\
\hline$\sigma_{7144}$ & 7288 & $\sigma_{7288}$ & 7325 & $\sigma_{7325}$ & 7337 & $\sigma_{7337}$ & 7758 & $\sigma_{7758}$ & 9074 & $\sigma_{9074}$ & \\
\hline 61 & 62 & 63 & 64 & 65 & 66 & 67 & 68 & 69 & 70 & 71 & \\
\hline 0.067 & 0.572 & 0.044 & 1.181 & 0.043 & 1.403 & 0.044 & 1.973 & 0.044 & 18.540 & 0.102 & \\
\hline 0.105 & 0.753 & 0.075 & 1.264 & 0.073 & 1.459 & 0.071 & 2.137 & 0.073 & 17.843 & 0.145 & \\
\hline 0.096 & 0.613 & 0.068 & 1.225 & 0.066 & 1.304 & 0.063 & 2.066 & 0.067 & 18.485 & 0.134 & \\
\hline$\ldots$ & $\cdots$ & $\ldots$ & $\ldots$ & $\ldots$ & $\ldots$ & $\cdots$ & $\cdots$ & $\cdots$ & $\ldots$ & $\ldots$ & \\
\hline
\end{tabular}

Notes. The $\mathrm{H} \beta$ fluxes are in units of $\mathrm{erg} \mathrm{s}^{-1} \mathrm{~cm}^{-2} \operatorname{arcsec}^{-2} . T(\mathrm{~S} 3)$ is the temperature derived from the $[\mathrm{S}$ III $] \lambda$ 9069/[S III] $\lambda$ 6312 ratio in a manner identical to that described for the [O III] temperature.

these maps. These maps are rotated $13^{\circ}$ with respect to the $\mathrm{N}-\mathrm{S}$ and $\mathrm{E}-\mathrm{W}$ directions, and are labeled with offsets in that rotated coordinate system. An outline of the area covered by these maps is shown in Figure 2.

Figure 7 shows the dereddened $\mathrm{H} \alpha$ surface brightness and demonstrates the effective resolution of the grid interpolated from the "data cube." It can be compared with the SOAR $\mathrm{H} \alpha$ image in Figure 1. Most features, including the bright arcs centered around NGC 2070, are visible. To the east the cavitylike region of low surface brightness as well as the bright rim on its eastern edge are clearly seen.

The ionization of the gas is traced by intensity ratios which include ([O III] $\lambda 5007) / \mathrm{H} \beta$ (Figure 9), ([N II] $\lambda 6584) / \mathrm{H} \alpha$ (Figure 10), ([S III] $\lambda 6312) /([\mathrm{S} \mathrm{II}] \lambda 6716+\lambda 6731)$ (Figure 11), and $\left[\mathrm{S}_{\mathrm{II}}\right] / \mathrm{H} \alpha$ (Figure 12). Figure 12 shows the ratio derived from the Blanco spectra, as opposed to the ratio made from the SOAR images that is shown in Figure 2. These line ratios

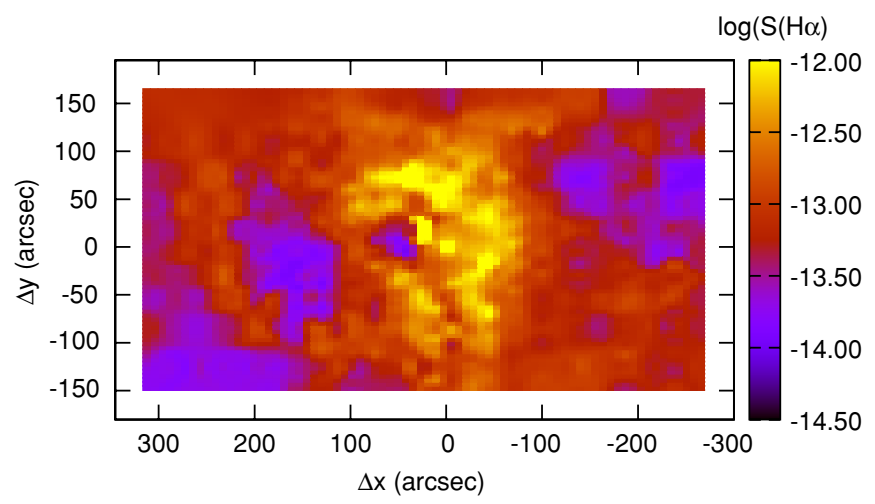

Figure 7. Interpolated dereddened $\mathrm{H} \alpha$ surface brightness in $\mathrm{erg} \mathrm{s}^{-1} \mathrm{~cm}^{-2}$ $\operatorname{arcsec}^{-2}$. The region shown is the same as that outlined in Figure 1 and Figures 8-12. R136 is at $\Delta x=0, \Delta y=0$.

(A color version of this figure is available in the online journal.) 
$A_{V}$

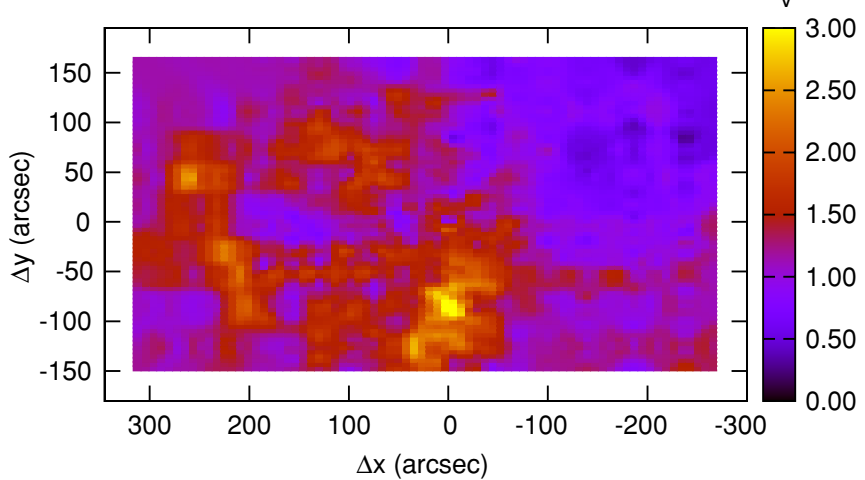

Figure 8. Interpolated $A_{V}$.

(A color version of this figure is available in the online journal.)

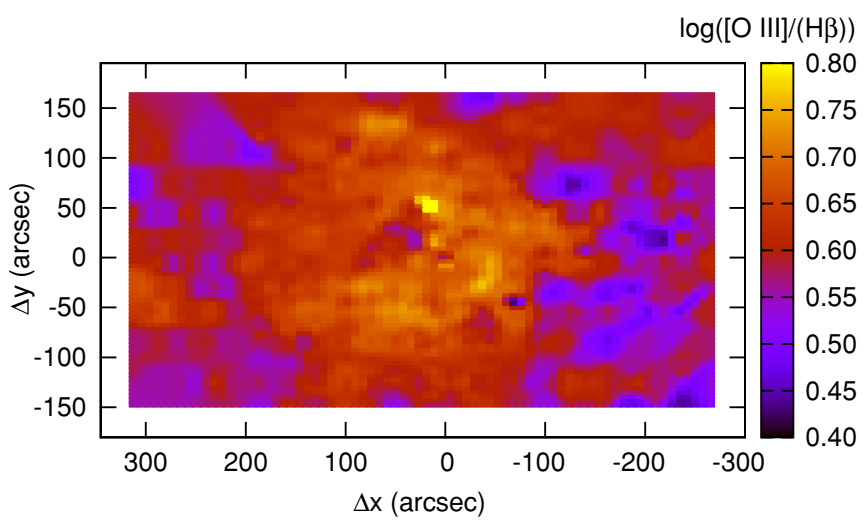

Figure 9. $\log ([\mathrm{O} \mathrm{III}] / \mathrm{H} \beta)$.

(A color version of this figure is available in the online journal.)

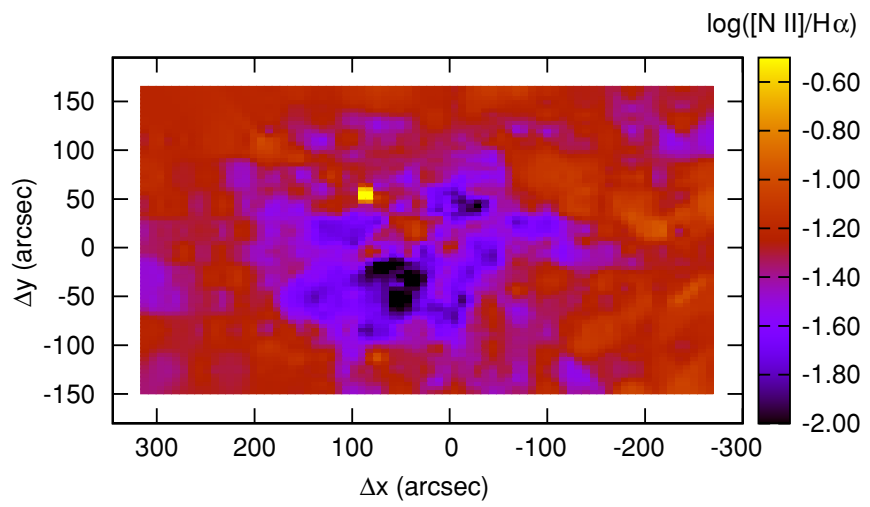

Figure 10. $\log (([\mathrm{N}$ II $] \lambda 6584) / \mathrm{H} \alpha)$

(A color version of this figure is available in the online journal.)

consistently show a rough circular symmetry around a point about $40 \operatorname{arcsec} \mathrm{E}$ of R136. The same distribution was identified by I09 from a Spitzer Space Telescope survey of 30 Dor using higher ionization lines. This will be discussed further below.

\section{2. [O III] Kinetic Temperature}

The electron kinetic temperature is computed from the [O III] $\lambda 4959+\lambda 5007 / \lambda 4363$ ratio. We find the nebula to be fairly isothermal within a few thousand degrees, in the range $9000 \mathrm{~K} \leqslant T_{e} \leqslant 12,000 \mathrm{~K}$, measured using the data points with nominal error bars smaller than $10 \%$.



Figure 11. $\log \left(\left[\mathrm{S}_{\mathrm{III}}\right] \lambda 6312 /\left(\left[\mathrm{S}_{\mathrm{II}}\right] \lambda 6716+\lambda 6731\right)\right)$.

(A color version of this figure is available in the online journal.)

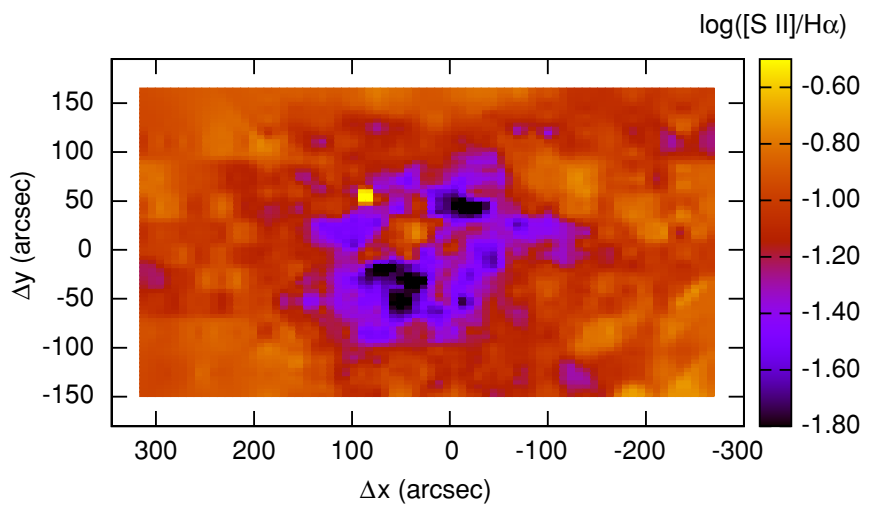

Figure 12. $\log \left(\left(\left[\mathrm{S}_{\mathrm{II}}\right] \lambda 6716+\lambda 6731\right) / \mathrm{H} \alpha\right)$.

(A color version of this figure is available in the online journal.)

The accuracy of the overall scale for the gas temperature will be an important point in our analysis in Paper II. Here we compare our mean [O III] temperature to that found by Krabbe \& Copetti (2002), who used three long-slit spectra which crisscrossed the central region of 30 Dor with much of their length falling on the bright arcs. There are a number of valid ways to calculate an average temperature $\left\langle T_{e}\right\rangle$ for 30 Dor. For the most direct comparison to distant, unresolved GEHRs, we should spatially integrate, over the whole nebula, all of the light in each emission line, and then apply a single reddening correction based on the ratio of the spatially integrated $\mathrm{H} \alpha$ and $\mathrm{H} \beta$ fluxes. From those reddening-corrected line strengths we can then find $\left\langle T_{e}\right\rangle$ using the observed [O III] $(\lambda 4959+\lambda 5007) / \lambda 4363$ ratio $R_{\mathrm{O} \text { III }}$ given by

$$
R_{\mathrm{OIII}}=\frac{\int F(4959 \AA+5007 \AA) d \Omega}{\int F(4363 \AA) d \Omega} .
$$

This method uses the total line fluxes listed for Position 38, row 3 in Table 4, and yields an equivalent temperature of $\left\langle T_{e}\right\rangle=10,680 \pm 5 \mathrm{~K}\left(R_{\mathrm{OIII}}=172 \pm 0.2\right)$. Here we have used the error bars on the measured line strengths derived as explained in the previous section.

An alternative is to use [O III] line fluxes that have first been individually dereddened at each point on the nebula and then spatially integrated. That method leads to $\left\langle T_{e}\right\rangle=10,760 \pm 8 \mathrm{~K}$ $\left(R_{\mathrm{O} \text { III }}=168 \pm 0.4\right)$. This corresponds to the values listed for Position 38, row 1 in Table 4.

When individual measurements of $T_{e}$ have been made at multiple locations in a nebula, as is the case here, another 
common approach is to compute

$$
\left\langle T_{e}\right\rangle=\frac{\int T_{e}(\vec{r}) \times F(H \beta) d \Omega}{\int F(H \beta) d \Omega},
$$

where $T_{e}$ is weighted by the $\mathrm{H} \beta$ flux at each position. Using this method we again find $\left\langle T_{e}\right\rangle=10,680 \mathrm{~K}$, equivalent to $R_{\mathrm{OII}}=$ 172.

For comparison, using this last method, Krabbe \& Copetti (2002) found $\left\langle T_{e}\right\rangle=10,270 \mathrm{~K}\left(R_{\mathrm{O} \text { III }}=195\right)$ using measurements along all three of their slit positions. An even lower $\left\langle T_{e}\right\rangle=$ $9990 \mathrm{~K}\left(R_{\mathrm{OIII}}=209\right)$ was found by Tsamis et al. (2003) who summed over the length of a 160 arcsec slit. Even though the formal standard error of the mean of each of these mean temperatures is $\sim 10 \mathrm{~K}$, the true uncertainties will be somewhat larger.

To explore the source of the discrepancy between our results and the previous measurements of $\left\langle T_{e}\right\rangle$, we coadded all of our Blanco spectra to produce a single spectrum with very high signal-to-noise ratio, excluding only the regions containing the brightest stars. The entire spectrum was then dereddened as in the first of the averaging methods described above. We then measured $R_{\mathrm{O} \text { III }}$ and computed the associated $\left\langle T_{e}\right\rangle$ in several ways.

First, using our automated line measuring software on this spectrum produced $\left\langle T_{e}\right\rangle=10,565 \mathrm{~K}\left(R_{\mathrm{OIII}}=178\right)$, in reasonable agreement with the value $\left\langle T_{e}\right\rangle=10,680 \mathrm{~K}$ obtained from the first of the averaging methods described above.

We then investigated the way in which our program sets the continuum level. The automated software fits the continuum in windows covering the wavelength ranges $\lambda \lambda 4280-4325$ (blueward of $\mathrm{H} \beta$ ) and $\lambda \lambda 4410-4150$ (redward of [O III] $\lambda 4363$ ). A manual measurement of the [O $\mathrm{III}]$ line strengths using the IRAF splot routine and similar continuum levels produced essentially the same $R_{\mathrm{O} \text { III }}$ and $\left\langle T_{e}\right\rangle$, verifying that our automated routine works correctly. However, inspection of the coadded spectrum showed that the region between $\mathrm{H} \beta$ and [O III] $\lambda 4363$ is partly filled in by a faint plateau due to line wings or weak emission, and that the continuum also has complications in the region around [O III] $\lambda 4959$ and $\lambda 5007$. We tried various choices of how to draw in the continuum and were able to measure line strengths corresponding to a temperature as low as $\left\langle T_{e}\right\rangle=$ $10,280 \mathrm{~K}\left(R_{\mathrm{OIII}}=194.6\right)$.

We conclude that the value $\left\langle T_{e}\right\rangle=10,270 \mathrm{~K}$ found by Krabbe $\&$ Copetti (2002) agrees with our corresponding value $\left\langle T_{e}\right\rangle=$ $10,673 \mathrm{~K}$ to within the measurement uncertainties due just to the issue of where the continuum is drawn. This uncertainty in the measured temperature translates to an uncertainty in the derived oxygen abundance. There would be a $12 \%$ ( 0.05 dex) increase in the derived $\mathrm{O} / \mathrm{H}$ abundance ratio if the temperature were decreased from $10,680 \mathrm{~K}$ to $10,280 \mathrm{~K}$. The $\lambda 5007 / \lambda 4363$ ratio measurements at individual points show a much larger scatter, with a standard deviation $\sigma=50 \%(0.29$ in the $\log$ ). Although this scatter is much larger than the uncertainty computed at each point from the photon counting statistics, we interpret it as being dominated by the true point-to-point measurement uncertainties.

We cannot resolve the discrepancy between the temperatures measured by Tsamis et al. (2003) and those measured here and by Krabbe \& Copetti (2002). However, we are confident that our measurements are correct to within the uncertainties discussed above.

\subsection{Ionization Mechanism}

The observed kinetic temperature provides an important constraint to the energy sources that ionize the gas. The violent history of 30 Doradus is evident in the diffuse X-ray emission that is present throughout the nebula (Townsley et al. 2006), as well as in the high-velocity expansion features with speeds up to $200 \mathrm{~km} \mathrm{~s}^{-1}$ seen in $\mathrm{H} \alpha$ emission (Chu \& Kennicutt 1994). These show that $\mathrm{SNe}$ and strong winds from massive $\mathrm{O}$ and WR stars have combined to heat gas to the observed $(3.5-7) \times 10^{6} \mathrm{~K}$ temperatures derived from Chandra X-ray spectra. Despite these indications of high-velocity flows, it has long been known that the moderate $10^{4} \mathrm{~K}$ temperatures in the ionized gas do not indicate shock heating as the current source of ionization.

The absence of [O IV] or [Ar V] emission lines (I09) as well as of any significant detection of nebular He II emission has been used to argue that strong shocks are not an important ionization mechanism in 30 Doradus. Instead, photoionization is implied as the energy input mechanism. However, this still leaves open the question of the source of the ionizing photons, which we now consider.

\section{STRUCTURAL DETAILS}

The geometry and ionization structure of 30 Dor are quite complex. The SOAR direct images provide a powerful tool for distinguishing edge-on IFs, regions ionized by localized sources of radiation besides the central cluster, optically thick pillars like those found in M16 (Hester et al. 1996), and line-emitting foreground structures. In particular, the nearly reddening-free [S II]/H $\alpha$ image (Figure 2), formed from the ratio of the SOAR images in those two passbands, offers considerable insight into the ionization structure on many scales.

\subsection{Embedded Ionizing Stars}

A key question is the degree to which the central cluster dominates the ionizing radiation field at different positions in the nebula. This will depend on the extent to which isolated $\mathrm{O}$ stars provide additional local contributions to the radiation field. Such stars can be recognized by localized, circular variations in the ionization level as indicated in the $[\mathrm{S}$ II $] / \mathrm{H} \alpha$ maps, in combination with brightness enhancements in the $\mathrm{H} \alpha$ emission. We carefully searched the SOAR images for such features, and located the 49 isolated stars listed in Table 6 . The spectral types of known stars within the affected region are listed, taken from Bosch et al. (2001), Parker \& Garmany (1993), Schaller et al. (1992), and Parker (1993) where available. Sources identified in previous surveys with unknown spectral types are listed as unknown and previously unidentified objects are left empty. Of the 49 sources identified, 19 have stars with known spectral types. There are four WR/WN, seven O, and six B-type stars. The later $\mathrm{O}$ and early B-type stars produce less ionizing radiation than more massive stars and may be illuminating some of the remnant material from their formation still present due to weak winds. The other objects identified with unknown spectral types are typically IR sources. Given the fraction of massive stars identified as embedded objects, the unknown sources should be followed up to determine if they too are massive stars. Some percentage of these is likely to have been selected by chance, so this estimate should represent an upper limit to the number of locally ionizing stars. The area of the nebula in which the ionization level is significantly affected by the radiation field of these stars, as projected on the sky, covers only $2 \%$ of the full 
Table 6

Regions of Possible Local Enhancement of Ionization Due to Nearby Massive Stars

\begin{tabular}{|c|c|c|c|c|c|c|c|}
\hline Object ID & R.A. & Decl. & $\begin{array}{c}\Delta \mathrm{R} . \mathrm{A} . \\
(\operatorname{arcsec})\end{array}$ & $\begin{array}{c}\Delta \text { decl. } \\
(\operatorname{arcsec})\end{array}$ & $\begin{array}{l}\text { Radius } \\
(\operatorname{arcsec})\end{array}$ & Spec. Type & Ref. \\
\hline 1 & 05:39:03.44 & $-69: 06: 35.72$ & 113 & -32 & 3.97 & $\mathrm{~B} 0.2 \mathrm{~V}^{\mathrm{a}}$ & \\
\hline 2 & $05: 39: 10.17$ & $-69: 06: 22.63$ & 149 & -19 & 3.97 & $\ldots$ & \\
\hline 3 & $05: 38: 57.10$ & $-69: 06: 06.62$ & 79 & -3 & 1.43 & $\mathrm{WN} 6 \mathrm{~h}+\mathrm{O}^{\mathrm{b}}$ & \\
\hline 4 & $05: 39: 05.43$ & $-69: 04: 16.27$ & 124 & 108 & 2.85 & O9.5III ${ }^{\mathrm{a}}$ & \\
\hline 5 & $05: 39: 05.51$ & $-69: 04: 31.61$ & 124 & 92 & 2.77 & O6.5III ${ }^{\mathrm{a}}$ & \\
\hline 6 & $05: 38: 24.46$ & $-69: 07: 57.28$ & -97 & -113 & 2.78 & $\ldots$ & \\
\hline 7 & $05: 38: 38.86$ & $-69: 08: 16.05$ & -19 & -132 & 4.76 & BN6 Iap ${ }^{c}$ & \\
\hline 8 & 05:38:01.61 & $-69: 04: 50.49$ & -219 & 73 & 1.69 & $\ldots$ & \\
\hline 9 & 05:38:01.31 & $-69: 04: 50.25$ & -221 & 74 & 1.35 & $\ldots$ & \\
\hline 10 & $05: 38: 41.19$ & $-69: 02: 57.75$ & -7 & 186 & 3.07 & $\mathrm{O} 7 \mathrm{~V}^{\mathrm{a}}$ & \\
\hline 11 & $05: 38: 45.39$ & $-69: 02: 50.84$ & 16 & 193 & 3.83 & $\mathrm{O} 4 \mathrm{~V}^{\mathrm{a}}$ & \\
\hline 12 & $05: 38: 13.98$ & $-69: 07: 47.63$ & -153 & -104 & 10.11 & Unkown & \\
\hline 13 & $05: 39: 20.55$ & $-69: 06: 54.42$ & 205 & -51 & 4.06 & $\ldots$ & \\
\hline 14 & $05: 39: 03.36$ & $-69: 09: 33.58$ & 113 & -210 & 3.02 & $\ldots$ & \\
\hline 15 & $05: 38: 45.08$ & $-69: 08: 08.06$ & 14 & -124 & 6.26 & $\mathrm{~B} 1 \mathrm{Ia}^{\mathrm{a}}+$ unknown & \\
\hline 16 & $05: 38: 52.82$ & $-69: 06: 12.01$ & 56 & -8 & 3.81 & $05.5 \mathrm{~V}^{\mathrm{a}}$ & \\
\hline 17 & $05: 38: 55.79$ & $-69: 05: 24.90$ & 72 & 39 & 2.41 & Unknown & \\
\hline 18 & $05: 38: 04.84$ & $-69: 07: 34.84$ & -202 & -91 & 3.54 & $\ldots$ & \\
\hline 19 & $05: 38: 31.75$ & $-69: 02: 14.28$ & -57 & 230 & 3.59 & Unknown & \\
\hline 20 & $05: 38: 41.76$ & $-69: 01: 58.79$ & -4 & 245 & 4.27 & Unknown & \\
\hline 21 & $05: 38: 53.54$ & $-69: 02: 00.56$ & 60 & 243 & 2.43 & $\mathrm{WN}^{\mathrm{d}}$ & \\
\hline 22 & $05: 39: 11.63$ & $-69: 02: 01.02$ & 157 & 243 & 3.07 & $\mathrm{WR}^{\mathrm{e}}$ & \\
\hline 23 & $05: 38: 15.64$ & $-69: 04: 37.64$ & -144 & 86 & 9.94 & Unknown & \\
\hline 24 & $05: 38: 54.94$ & $-69: 08: 45.76$ & 67 & -162 & 3.77 & B0.5 $\mathrm{Ia}^{\mathrm{a}}$ & \\
\hline 25 & $05: 38: 56.83$ & $-69: 08: 42.47$ & 77 & -159 & 4.25 & A2/3Ia ${ }^{a}+$ unknown & \\
\hline 26 & $05: 38: 54.70$ & $-69: 07: 45.81$ & 66 & -102 & 5.29 & G8V ${ }^{a}$ & \\
\hline 27 & $05: 38: 36.43$ & $-69: 06: 58.70$ & -32 & -55 & 2.98 & $\mathrm{WN}^{\mathrm{e}}$ & \\
\hline 28 & $05: 38: 36.06$ & $-69: 06: 47.52$ & -34 & -44 & 2.04 & $\mathrm{O} 8.5 \mathrm{~V}^{\mathrm{a}}+\mathrm{B} 0.5 \mathrm{III}^{\mathrm{a}}+\mathrm{B} 1 \mathrm{Ia}^{\mathrm{b}}$ & \\
\hline 29 & $05: 38: 51.32$ & $-69: 06: 41.99$ & 48 & -38 & 2.84 & Unknown & \\
\hline 30 & $05: 38: 49.79$ & $-69: 06: 44.12$ & 40 & -40 & 1.7 & $\mathrm{~B} 0.5 \mathrm{I}^{\mathrm{a}}$ & \\
\hline 31 & $05: 38: 57.40$ & $-69: 07: 10.75$ & 81 & -67 & 2.73 & $\mathrm{~B} 3 \mathrm{Ia}^{\mathrm{a}}$ & \\
\hline 32 & $05: 38: 46.60$ & $-69: 04: 28.07$ & 22 & 96 & 2.3 & $\mathrm{O} 4 \mathrm{~V}^{\mathrm{a}}$ & \\
\hline 33 & $05: 38: 36.91$ & $-69: 05: 08.19$ & -30 & 56 & 5.97 & $\ldots$ & \\
\hline 34 & 05:39:01.05 & $-69: 06: 30.16$ & 100 & -26 & 2.44 & $\ldots$ & \\
\hline 35 & $05: 38: 58.80$ & $-69: 05: 24.55$ & 88 & 39 & 2.46 & $\ldots$ & \\
\hline 36 & $05: 38: 17.62$ & $-69: 05: 42.83$ & -133 & 21 & 13.67 & $\ldots$ & \\
\hline 37 & $05: 39: 22.86$ & $-69: 07: 46.88$ & 217 & -103 & 3.39 & $\ldots$ & \\
\hline 38 & $05: 39: 12.35$ & $-69: 06: 02.74$ & 161 & 1 & 4.78 & $\ldots$ & \\
\hline 39 & 05:38:14.88 & $-69: 04: 31.80$ & -148 & 92 & 2.31 & $\ldots$ & \\
\hline 40 & $05: 38: 08.53$ & $-69: 05: 44.36$ & -182 & 19 & 15.11 & $\ldots$ & \\
\hline 41 & $05: 38: 10.64$ & $-69: 06: 17.52$ & -171 & -14 & 3.69 & $\ldots$ & \\
\hline 42 & 05:38:09.46 & $-69: 06: 22.26$ & -177 & -18 & 3.69 & $\ldots$ & \\
\hline 43 & $05: 37: 49.22$ & $-69: 06: 14.27$ & -286 & -10 & 4.41 & $\ldots$ & \\
\hline 44 & $05: 38: 24.70$ & $-69: 07: 43.55$ & -95 & -100 & 3.06 & $\ldots$ & \\
\hline 45 & $05: 38: 30.51$ & $-69: 06: 46.21$ & -64 & -42 & 3.07 & $\ldots$ & \\
\hline 46 & $05: 38: 29.73$ & $-69: 06: 57.41$ & -68 & -54 & 3.07 & $\ldots$ & \\
\hline 47 & 05:38:31.02 & $-69: 06: 37.47$ & -61 & -34 & 2.63 & $\ldots$ & \\
\hline
\end{tabular}

Notes. Stellar spectral types are from

a Bosch et al. 1999.

b Schnurr et al. 2009.

c Walborn \& Blades 1997.

${ }^{\mathrm{d}}$ Robert et al. 2003.

e Breysacher 1981. Objects found in surveys with no reported spectral type are listed as unknown. Objects with no known counterpart are left blank.

nebula. This means that it will not be an important contaminant in our analysis, in Paper II, of the overall properties of the nebula.

\subsection{Dense Pillars}

There are numerous bright fingers and protruding IFs scattered over the face of the nebula, but they are particularly concentrated in the large X-ray emitting cavity to the east of R136.
Many of these are probably elephant trunks similar to the famous "Pillars of Creation" in M16. A small number of these have been commented on by Scowen et al. (1998). These structures are easily identified using a combination of the $\mathrm{H} \alpha$, [S II] and $8 \mu \mathrm{m}$ images. Table 7 catalogs 106 of the brightest such features, listing their RA and Dec positions, projected length and P.A. However, there are hundreds more that blend together as one looks on finer and finer scales, until they are indistinguish- 
Table 7

A Catalog of Bright, Dense Pillars and Protruding IFs

\begin{tabular}{|c|c|c|c|c|c|c|}
\hline Pillar ID & R.A. (J2000) & Decl. & $\begin{array}{c}\Delta \mathrm{R} . \mathrm{A} . \\
(\operatorname{arcsec})\end{array}$ & $\begin{array}{l}\Delta \mathrm{Decl} . \\
(\operatorname{arcsec})\end{array}$ & $\begin{array}{l}\text { Length } \\
(\operatorname{arcsec})\end{array}$ & P.A \\
\hline 1 & $05: 37: 28.35$ & $-69: 01: 51.86$ & -400 & 252 & 37 & 216 \\
\hline 2 & $05: 37: 30.68$ & $-69: 04: 12.44$ & -384 & 111 & 17 & 120 \\
\hline 3 & $05: 37: 32.85$ & $-69: 04: 16.79$ & -373 & 107 & 19 & 115 \\
\hline 4 & $05: 37: 42.14$ & $-69: 02: 30.70$ & -325 & 213 & 26 & 209 \\
\hline 5 & $05: 37: 42.85$ & $-69: 04: 42.79$ & -319 & 81 & 10 & 176 \\
\hline 6 & $05: 37: 42.95$ & $-69: 04: 34.28$ & -319 & 90 & 10 & 183 \\
\hline 7 & $05: 37: 50.28$ & $-69: 06: 02.92$ & -282 & 1 & 19 & 155 \\
\hline 8 & $05: 37: 54.67$ & $-69: 05: 09.56$ & -255 & 54 & 9 & 167 \\
\hline 9 & $05: 37: 58.57$ & $-69: 03: 44.17$ & -233 & 140 & 7 & 182 \\
\hline 10 & 05:37:59.29 & $-69: 05: 49.22$ & -233 & 15 & 8 & 163 \\
\hline 11 & 05:38:03.81 & $-69: 05: 22.05$ & -207 & 42 & 10 & 164 \\
\hline 12 & 05:38:05.74 & $-69: 06: 36.40$ & -196 & -33 & 6 & 138 \\
\hline 13 & 05:38:08.17 & $-69: 06: 51.16$ & -185 & -47 & 33 & 167 \\
\hline 14 & 05:38:09.49 & $-69: 07: 05.29$ & -180 & -61 & 19 & 159 \\
\hline 15 & $05: 38: 11.95$ & $-69: 07: 08.39$ & -164 & -65 & 26 & 142 \\
\hline 16 & 05:38:13.19 & $-69: 04: 25.76$ & -158 & 98 & 13 & 236 \\
\hline 17 & $05: 38: 14.22$ & $-69: 07: 00.28$ & -153 & -56 & 10 & 131 \\
\hline 18 & 05:38:15.89 & $-69: 05: 25.19$ & -142 & 39 & 6 & 260 \\
\hline 19 & $05: 38: 17.03$ & $-69: 05: 57.00$ & -137 & 7 & 3 & 112 \\
\hline 20 & $05: 38: 17.56$ & $-69: 05: 02.66$ & -131 & 61 & 14 & 200 \\
\hline 21 & $05: 38: 24.12$ & $-69: 04: 56.88$ & -99 & 67 & 24 & 206 \\
\hline 22 & $05: 38: 28.52$ & $-69: 08: 26.14$ & -72 & -142 & 16 & 166 \\
\hline 23 & $05: 38: 28.74$ & $-69: 03: 37.29$ & -72 & 147 & 6 & 238 \\
\hline 24 & $05: 38: 30.07$ & $-69: 08: 38.73$ & -67 & -155 & 24 & 138 \\
\hline 25 & $05: 38: 33.14$ & $-69: 06: 02.88$ & -51 & 1 & 5 & 153 \\
\hline 26 & $05: 38: 34.01$ & $-69: 03: 33.01$ & -45 & 151 & 12 & 268 \\
\hline 27 & $05: 38: 34.26$ & $-69: 06: 26.96$ & -45 & -23 & 12 & 153 \\
\hline 28 & $05: 38: 36.05$ & $-69: 07: 43.52$ & -35 & -100 & 12 & 148 \\
\hline 29 & $05: 38: 38.27$ & $-69: 08: 13.16$ & -24 & -129 & 9 & 81 \\
\hline 30 & $05: 38: 40.51$ & $-69: 09: 58.77$ & -8 & -235 & 9 & 111 \\
\hline 31 & 05:38:40.97 & $-69: 07: 27.27$ & -8 & -83 & 12 & 84 \\
\hline 32 & $05: 38: 43.16$ & $-69: 07: 04.03$ & 3 & -60 & 7 & 96 \\
\hline 33 & $05: 38: 43.20$ & $-69: 01: 19.02$ & 3 & 285 & 15 & 173 \\
\hline 34 & $05: 38: 43.23$ & $-69: 10: 21.48$ & 3 & -258 & 11 & 132 \\
\hline 35 & $05: 38: 44.16$ & $-69: 06: 58.89$ & 8 & -55 & 5 & 90 \\
\hline 36 & $05: 38: 44.25$ & $-69: 11: 10.68$ & 8 & -307 & 9 & 127 \\
\hline 37 & $05: 38: 45.18$ & $-69: 05: 05.74$ & 14 & 58 & 8 & 304 \\
\hline 38 & $05: 38: 45.41$ & $-69: 04: 19.41$ & 14 & 104 & 8 & 268 \\
\hline 39 & $05: 38: 45.65$ & $-69: 10: 25.07$ & 19 & -261 & 18 & 108 \\
\hline 40 & $05: 38: 45.85$ & $-69: 09: 51.28$ & 19 & -227 & 15 & 62 \\
\hline 41 & $05: 38: 46.15$ & $-69: 07: 04.11$ & 19 & -60 & 8 & 86 \\
\hline 42 & $05: 38: 46.86$ & $-69: 09: 33.58$ & 25 & -210 & 7 & 73 \\
\hline 43 & 05:38:47.05 & $-69: 07: 56.66$ & 25 & -113 & 13 & 61 \\
\hline 44 & $05: 38: 47.85$ & $-69: 07: 15.15$ & 30 & -71 & 14 & 65 \\
\hline 45 & $05: 38: 50.55$ & $-69: 10: 17.26$ & 46 & -253 & 16 & 77 \\
\hline 46 & $05: 38: 53.45$ & $-69: 08: 00.23$ & 57 & -116 & 8 & 70 \\
\hline 47 & $05: 38: 54.71$ & $-69: 05: 36.11$ & 68 & 28 & 3 & 343 \\
\hline 48 & $05: 38: 55.01$ & $-69: 05: 39.94$ & 68 & 24 & 3 & 309 \\
\hline 49 & 05:38:55.29 & $-69: 05: 40.63$ & 68 & 23 & 3 & 267 \\
\hline 50 & $05: 38: 56.40$ & $-69: 07: 21.25$ & 73 & -77 & 13 & 30 \\
\hline 51 & $05: 38: 56.81$ & $-69: 05: 30.58$ & 78 & 33 & 10 & 297 \\
\hline 52 & 05:38:57.29 & $-69: 01: 56.18$ & 78 & 248 & 5 & 32 \\
\hline 53 & $05: 38: 57.51$ & $-69: 06: 27.65$ & 84 & -24 & 16 & 16 \\
\hline 54 & $05: 38: 57.81$ & $-69: 07: 56.53$ & 84 & -113 & 19 & 68 \\
\hline 55 & 05:38:58.07 & $-69: 01: 51.67$ & 84 & 252 & 7 & 23 \\
\hline 56 & $05: 38: 58.58$ & $-69: 02: 16.27$ & 89 & 228 & 9 & 233 \\
\hline 57 & $05: 38: 58.84$ & $-69: 07: 28.60$ & 89 & -85 & 15 & 43 \\
\hline 58 & $05: 38: 59.48$ & $-69: 08: 04.34$ & 89 & -121 & 37 & 67 \\
\hline 59 & 05:39:00.01 & $-69: 11: 45.42$ & 95 & -342 & 22 & 83 \\
\hline 60 & 05:39:00.14 & $-69: 06: 08.15$ & 95 & -4 & 6 & 340 \\
\hline 61 & 05:39:00.29 & $-69: 08: 56.01$ & 95 & -172 & 21 & 52 \\
\hline 62 & $05: 39: 02.13$ & $-69: 08: 22.12$ & 105 & -138 & 17 & 46 \\
\hline 63 & 05:39:02.47 & $-69: 06: 40.10$ & 105 & -36 & 17 & 29 \\
\hline 64 & 05:39:03.49 & $-69: 07: 31.02$ & 111 & -87 & 19 & 33 \\
\hline 65 & $05: 39: 04.13$ & $-69: 08: 14.74$ & 116 & -131 & 23 & 57 \\
\hline
\end{tabular}

Table 7

(Continued)

\begin{tabular}{|c|c|c|c|c|c|c|}
\hline Pillar ID & R.A. (J2000) & Decl. & $\begin{array}{c}\Delta \text { R.A. } \\
(\operatorname{arcsec})\end{array}$ & $\begin{array}{l}\Delta \text { Decl. } \\
(\operatorname{arcsec})\end{array}$ & $\begin{array}{l}\text { Length } \\
(\operatorname{arcsec})\end{array}$ & P.A. \\
\hline 66 & 05:39:05.07 & $-69: 05: 54.83$ & 121 & 9 & 7 & 279 \\
\hline 67 & 05:39:07.00 & $-69: 10: 49.34$ & 132 & -286 & 7 & 39 \\
\hline 68 & 05:39:07.51 & $-69: 10: 26.01$ & 138 & -262 & 14 & 12 \\
\hline 69 & 05:39:08.05 & $-69: 08: 47.38$ & 138 & -164 & 18 & 53 \\
\hline 70 & $05: 39: 11.46$ & $-69: 08: 15.27$ & 154 & -131 & 9 & 49 \\
\hline 71 & $05: 39: 11.76$ & $-69: 10: 53.42$ & 159 & -290 & 14 & 55 \\
\hline 72 & $05: 39: 12.36$ & $-69: 07: 44.75$ & 159 & -101 & 13 & 26 \\
\hline 73 & $05: 39: 13.25$ & $-69: 08: 56.22$ & 164 & -172 & 9 & 91 \\
\hline 74 & $05: 39: 14.28$ & $-69: 01: 45.16$ & 170 & 259 & 23 & 308 \\
\hline 75 & $05: 39: 14.63$ & $-69: 10: 54.29$ & 175 & -290 & 24 & 54 \\
\hline 76 & $05: 39: 14.72$ & $-69: 07: 39.20$ & 175 & -95 & 13 & 37 \\
\hline 77 & $05: 39: 16.70$ & $-69: 05: 28.48$ & 186 & 35 & 8 & 350 \\
\hline 78 & 05:39:17.98 & $-69: 10: 36.02$ & 191 & -272 & 11 & 32 \\
\hline 79 & 05:39:18.95 & $-69: 05: 56.61$ & 197 & 7 & 11 & 355 \\
\hline 80 & 05:39:18.97 & $-69: 07: 45.44$ & 197 & -102 & 8 & 63 \\
\hline 81 & 05:39:19.05 & $-69: 07: 45.59$ & 197 & -102 & 8 & 55 \\
\hline 82 & $05: 39: 21.91$ & $-69: 04: 31.65$ & 213 & 92 & 15 & 335 \\
\hline 83 & $05: 39: 22.10$ & $-69: 05: 27.13$ & 213 & 37 & 18 & 341 \\
\hline 84 & $05: 39: 22.91$ & $-69: 06: 08.13$ & 218 & -4 & 7 & 359 \\
\hline 85 & $05: 39: 23.79$ & $-69: 07: 00.26$ & 224 & -56 & 24 & 14 \\
\hline 86 & $05: 39: 24.53$ & $-69: 04: 45.88$ & 229 & 78 & 9 & 339 \\
\hline 87 & $05: 39: 26.37$ & $-69: 05: 59.02$ & 234 & 5 & 15 & 27 \\
\hline 88 & $05: 39: 26.59$ & $-69: 04: 42.32$ & 240 & 81 & 4 & 344 \\
\hline 89 & $05: 39: 26.62$ & $-69: 07: 32.13$ & 240 & -88 & 22 & 28 \\
\hline 90 & $05: 39: 27.52$ & $-69: 06: 11.28$ & 245 & -7 & 9 & 349 \\
\hline 91 & $05: 39: 27.79$ & $-69: 04: 04.10$ & 245 & 120 & 28 & 320 \\
\hline 92 & $05: 39: 30.83$ & $-69: 05: 55.85$ & 261 & 8 & 20 & 25 \\
\hline 93 & $05: 39: 30.85$ & $-69: 10: 11.75$ & 261 & -248 & 19 & 44 \\
\hline 94 & 05:39:31.05 & $-69: 05: 29.34$ & 261 & 34 & 8 & 341 \\
\hline 95 & $05: 39: 31.14$ & $-69: 05: 24.43$ & 261 & 39 & 6 & 330 \\
\hline 96 & $05: 39: 31.23$ & $-69: 05: 20.09$ & 261 & 44 & 16 & 357 \\
\hline 97 & $05: 39: 34.55$ & $-69: 05: 25.43$ & 283 & 38 & 6 & 324 \\
\hline 98 & $05: 39: 37.13$ & $-69: 07: 28.57$ & 293 & -85 & 13 & 12 \\
\hline 99 & $05: 39: 37.64$ & $-69: 07: 19.20$ & 299 & -75 & 10 & 13 \\
\hline 100 & $05: 39: 37.86$ & $-69: 07: 09.53$ & 299 & -66 & 8 & 8 \\
\hline 101 & $05: 39: 39.19$ & $-69: 11: 25.06$ & 304 & -321 & 7 & 240 \\
\hline 102 & $05: 39: 39.53$ & $-69: 08: 34.63$ & 310 & -151 & 5 & 29 \\
\hline 103 & $05: 39: 39.73$ & $-69: 08: 31.87$ & 310 & -148 & 4 & 58 \\
\hline 104 & $05: 39: 39.77$ & $-69: 11: 25.05$ & 310 & -321 & 12 & 258 \\
\hline 105 & 05:39:44.91 & $-69: 06: 49.21$ & 336 & -45 & 17 & 2 \\
\hline 106 & $05: 39: 47.81$ & $-69: 08: 49.79$ & 353 & -166 & 39 & 19 \\
\hline
\end{tabular}

able from small examples of the edge-on IFs discussed above. Almost all of these point back to R136, strongly suggesting that the central cluster is the dominant source of ionizing radiation.

Many of these features are clearly connected to larger bodies of molecular gas, as can be seen by comparing our $[\mathrm{S} \mathrm{II}] / \mathrm{H} \alpha$ ratio image to the $8 \mu \mathrm{m}$ image from the Spitzer Space Telescope archives, which shows PAH emission (Figure 13). However, some of them do appear to be isolated tubes or blobs of gas rather than protrusions from background walls of molecular material. Scowen et al. (1998) have commented on one bright structure of this latter type. Some of these might correspond to the expanding shells seen in $\mathrm{H} \alpha$ emission in the high-resolution echelle spectra of Chu \& Kennicutt (1994).

\subsection{Edge-on Ionization Fronts and the Source of the Ionizing Radiation}

Edge-on IFs stand out in the $[\mathrm{S} \mathrm{II}] / \mathrm{H} \alpha$ image as narrow linear structures with high $[\mathrm{S} \mathrm{II}] / \mathrm{H} \alpha$ ratio, butted up against regions of low $[\mathrm{S} \mathrm{II}] / \mathrm{H} \alpha$. Table 8 lists prominent, isolated, 

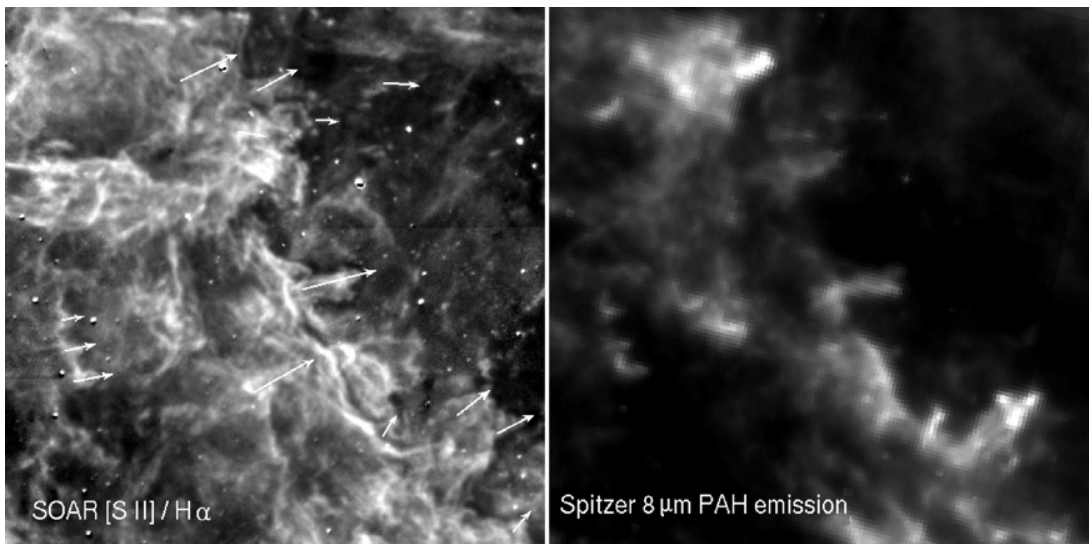

Figure 13. Left SOAR [S II]/H $\alpha$; Right Spitzer $8 \mu \mathrm{m}$ PAH. A selection of bright pillars is shown with arrows indicating their location and direction. These dense IFs are detected in both optical and IR passbands and show a connection with the background molecular cloud.

edge-on IFs found both in our $[\mathrm{S} \mathrm{II}] / \mathrm{H} \alpha$ image and in the Spitzer $8 \mu \mathrm{m}$ PAH mosaic. The structures identified in both the optical and IR passbands are perfectly suited for detailed, high angular resolution (e.g., with HST and/or ALMA) studies of the neutral and molecular gas beyond the IF, commonly called the photo-dissociation region or photon-dominated region (PDR). A finding chart of all IFs listed in Table 8 is shown on a [S II]/ $\mathrm{H} \alpha$ image in Figure 14.

With our new observations there is enough information available about several of these IFs to test whether or not they are likely to be photoionized by the central cluster including $\mathrm{R} 136$. The thickness of the ionized gas layer $d r$, depends on the density $n$ and the distance of the gas from the source of ionizing radiation $r_{0}$ according to

$$
d r \propto \frac{Q_{0}}{4 \pi \alpha n_{e} n_{p} r_{0}^{2}},
$$

where $Q_{0}$ is the ionizing photon luminosity by number, $\alpha=$ $2.59 \times 10^{-13} \mathrm{~cm}^{-3} \mathrm{~s}^{-1}$ is the $\mathrm{H}^{+}$recombination rate, and we assume $d r \ll r_{0}$. The test consists of computing $Q_{0}$ values for different IFs at very different distances from R136, assuming that R136 marks the center of the ionizing radiation, and seeing if the different $Q_{0}$ measurements agree with each other. There will be considerable uncertainty due to the implicit assumption that the projected distance from R136 to each IF is the true threedimensional distance, so we do not carry error bars through the analysis and will regard agreement at a factor of 2 level to be satisfactory.

Two IFs of interest for this test, IF1 and IF2, are fairly close to 30 Dor (see Figure 14). Both have a spatially resolved layer of $\mathrm{H}^{+}$separated from an edge-on IF seen in [S II], similar to the Orion Bar (see Pellegrini et al. 2009).

In the case of IF1, there is a line of stars coincident with the IF that could be an alternative to NGC 2070 as the source of ionization, or which could be stars formed in a region of gas that was compressed by radiation and wind pressure from the cluster, or which could simply be foreground stars. Most of these stars fell in one or another of our slit positions. To gauge the influence these stars may have on the ionization structure of this region, we have examined the stellar spectra and found them to lack any absorption features. This would indicate they are massive stars; however this result is uncertain due to the brightness of the emission lines coincident with the expected absorption features. If these stars were embedded in the gas we
Table 8

Prominent Ionization Fronts (IFs) Suitable for Follow-up Multi-wavelength Studies

\begin{tabular}{|c|c|c|c|c|c|c|}
\hline IF ID & R.A. (J2000) & Decl. & $\begin{array}{c}\Delta \text { R.A. } \\
(\operatorname{arcsec})\end{array}$ & $\begin{array}{l}\Delta \text { Decl. } \\
(\operatorname{arcsec})\end{array}$ & $\begin{array}{l}\text { Length } \\
(\operatorname{arcsec})\end{array}$ & P.A. \\
\hline 1 & $05: 38: 44.15$ & $-69: 06: 59.71$ & 9.3 & -55.9 & 31.0 & 257.7 \\
\hline 2 & 05:38:55.02 & $-69: 05: 42.02$ & 67.7 & 21.8 & & 317.9 \\
\hline 3 & 05:38:36.07 & $-69: 06: 27.99$ & -34.1 & -24.2 & & 349.1 \\
\hline 4 & $05: 39: 22.75$ & $-69: 07: 17.56$ & 216.8 & -73.8 & & 34.7 \\
\hline 5 & 05:39:11.80 & $-69: 08: 14.33$ & 157.9 & -130.5 & & 68.9 \\
\hline 6 & 05:38:59.15 & $-69: 05: 14.79$ & 89.9 & & & 120.0 \\
\hline 7 & $05: 38: 51.70$ & $-69: 05: 06.06$ & 49.9 & 57.7 & & 70.0 \\
\hline 8 & 05:37:55.71 & $-69: 05: 43.70$ & -251.1 & 20.1 & & 321.9 \\
\hline 9 & 7:54.88 & $-69: 05: 11.42$ & -255.6 & 52.4 & & 306.1 \\
\hline 10 & 34 & $-69: 05:$ & -306.8 & & & 0.0 \\
\hline 11 & .22 & $-69: 07: 4$ & -194.6 & -99.7 & & 344.1 \\
\hline 12 & 1.38 & $-69: 08: 0$ & -166.9 & -122.6 & & 283.8 \\
\hline 13 & & $-69: 07: 1$ & 295.1 & -73.7 & & 26.0 \\
\hline 14 & & $-69: 08: 2$ & 296.5 & -141.4 & & 63.0 \\
\hline 15 & 05:39:23.05 & $-69: 08: 33.57$ & 218.4 & -149.8 & & 310.0 \\
\hline 16 & 05:39:19.76 & $-69: 08: 2$ & 200.7 & -137.2 & & 29.2 \\
\hline 17 & $5: 38: 58.60$ & $-69: 09: 3$ & 87.0 & -21 & & 16.9 \\
\hline 18 & $5: 38: 32.82$ & $-69: 09: 17.55$ & -51.6 & -193.8 & & 340.7 \\
\hline 19 & $: 38: 58.56$ & $-69: 08: 43.34$ & 86.8 & -159.5 & 2.4 & 324.7 \\
\hline 20 & 05:39:03.35 & $-69: 08: 04.44$ & 112.5 & -120.6 & & 67.9 \\
\hline 21 & .79 & $-69: 04:$ & 147.1 & 87.2 & & 142.7 \\
\hline 22 & 2.60 & $-69: 04:$ & 54 & 83.5 & 2 & 128.7 \\
\hline 23 & & $-69:($ & 12. & 104.5 & 2 & 120.0 \\
\hline 24 & 7.67 & $-69: 04$ & -25.5 & 96.2 & 3.6 & 45.0 \\
\hline 25 & & $-69: 03$ & -17 & 152.1 & & 90.0 \\
\hline 26 & 05:38:28.95 & $-69: 02: 54.60$ & -72.4 & & & 25.7 \\
\hline 27 & $05: 38: 23.40$ & $-69: 02: 24.23$ & -102.2 & 219.6 & 62.5 & 111.4 \\
\hline 28 & & $-69: 02: 16.68$ & -160.4 & 227.1 & & 66.2 \\
\hline 29 & 05:38:10.17 & $-69: 02: 44.58$ & -173.4 & 199.2 & 11.4 & 61.0 \\
\hline 30 & 05:38:07.60 & $-69: 03: 16.01$ & -187.2 & 167.8 & 15.7 & 39.1 \\
\hline 31 & $05: 38: 14.24$ & $-69: 03: 29.95$ & -151.5 & 153.8 & & 53.0 \\
\hline 32 & $05: 38: 38.16$ & $-69: 00: 34.55$ & -22 & 329.2 & 28.8 & 63.8 \\
\hline 33 & 05:38:04.92 & $-69: 04: 38.91$ & -201.6 & 84.9 & 9.2 & 26.3 \\
\hline 34 & 05:38:34.69 & $-69: 01: 05.06$ & -41.6 & 298.7 & 20.8 & 32.5 \\
\hline & & $-69: 07: 44.65$ & -241 & -100.9 & 107.0 & 322.2 \\
\hline $2 *$ & 05:38:09.50 & $-69: 08: 58.47$ & -177.0 & -174.7 & 83.8 & 298.5 \\
\hline $3^{*}$ & 05:38:19.48 & $-69: 09: 55.73$ & -123.3 & -231.9 & 54.8 & 343.7 \\
\hline $4 *$ & 05:38:06.23 & $-69: 10: 47.75$ & -194.5 & -284.0 & 54.8 & 60.8 \\
\hline & $05: 37: 58.58$ & $-69: 08: 32.56$ & -235.7 & -148.8 & 18.4 & 317.2 \\
\hline
\end{tabular}

Notes. IDs with an asterisk identify IFs with PAH emission closer to R136 than the $[\mathrm{S}$ II] emission which indicate an ionization source other than the central cluster. 


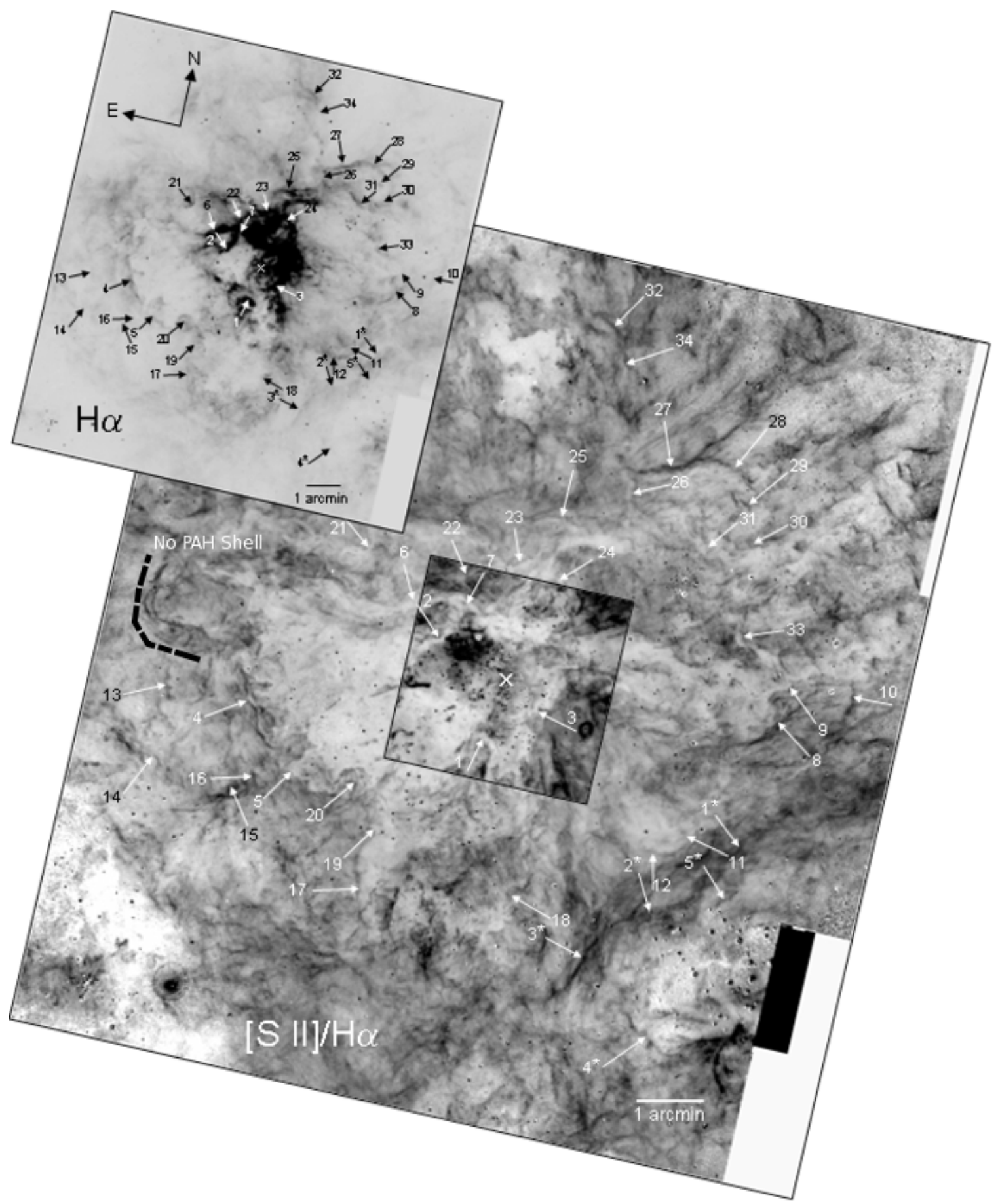

Figure 14. $\mathrm{H} \alpha$ (upper) and $[\mathrm{S} \mathrm{II}] / \mathrm{H} \alpha$ (lower) images showing the positions of the ionization fronts (IFs) cataloged in Table 8. The approximate center of each IF is at the tip of the arrow marking it. The arrows point in the direction toward higher ionization within each I-front, and except for IF16 are approximately perpendicular to the IF. On the main image, darker shades indicate larger values of [S II]/H $\alpha$, but with the gray scale adjusted in the central box to show regions with lower [S II]/H $\alpha$ ratios. The white $\mathrm{X}$ in each image marks the position of $\mathrm{R} 136$.

would expect the stellar spectra to be significantly reddened, but with the possible exception of one star, this does not appear to be the case. Additionally, we note that the overall structure of IF1 is oriented almost exactly perpendicular to the direction to R136. There are also many prominent pillars in this region, all pointing back up to R136, suggesting the nearby stars are unimportant to the structure.

Our SOAR spectrographic slit position number 40 cuts directly across IF1, avoiding the pillars noted by Scowen et al. (1998) and targeting the larger structure. The emission-line strengths in the region of IF1 were measured as described above, with the exception that the extraction window is smaller ( 0.75 arcsec) along the slit to better resolve the detailed structure. Figure 15 shows the clear separation of the highly ionized gas from the lower-ionization gas in IF1. In the top panel are the relative intensities of $\mathrm{H} \alpha$, [O III] $\lambda 5007$, and [S III] $\lambda 9069$ which trace highly ionized gas. The middle panel shows the same for $\left[\mathrm{S}_{\mathrm{II}}\right],\left[\mathrm{O}_{\mathrm{II}}\right]$, and $\left[\mathrm{N}_{\mathrm{II}}\right]$, all tracers of the IF. The bottom panel shows the linear density profile of the structure. At the illuminated face the structure has an electron density 

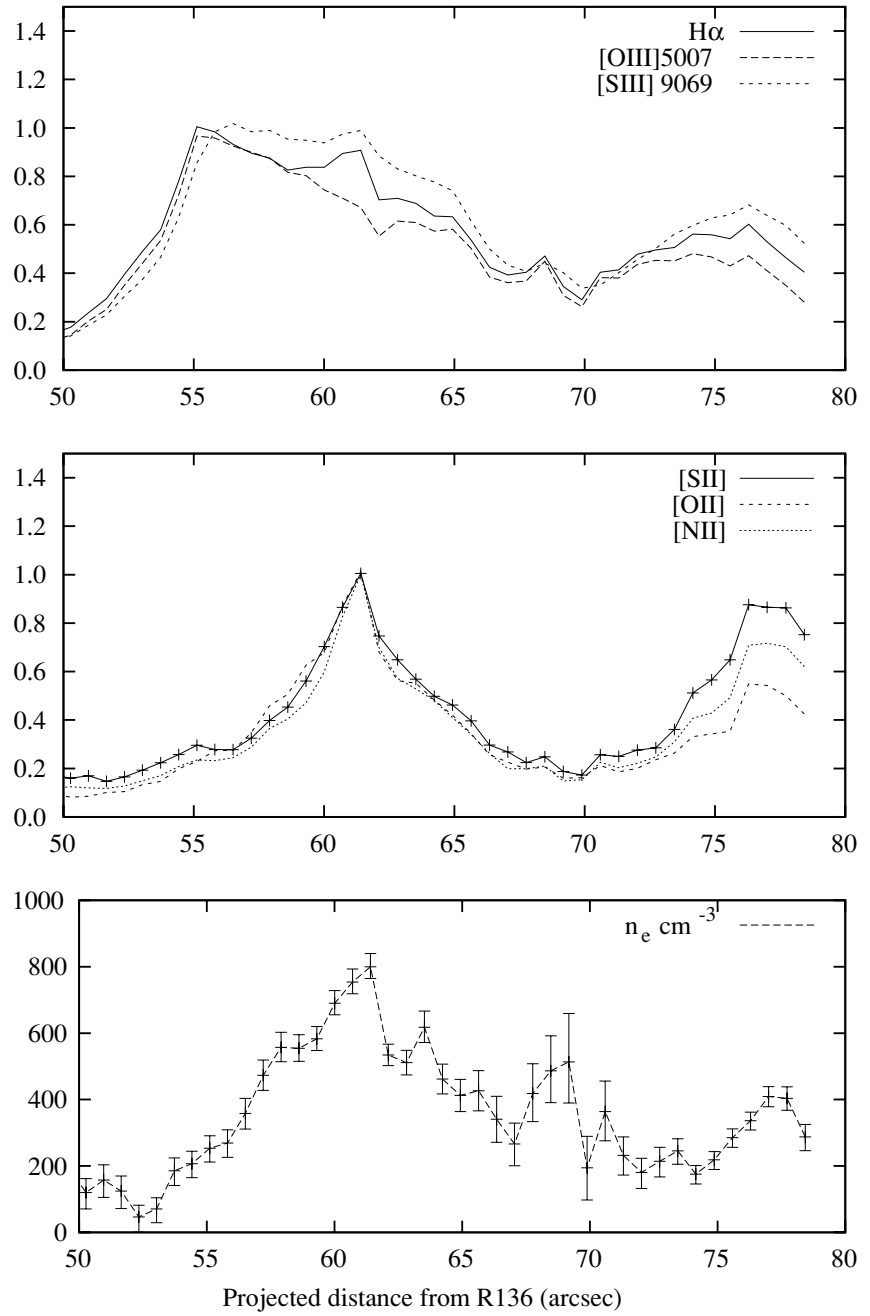

Figure 15. Profile of IF1. Top: ionized gas is traced by $\mathrm{H} \alpha$, [O III], and [S III] emission. Middle: the IF is traced by [S II], [N II] and [O II]. Bottom: the electron density profile in $\mathrm{cm}^{-3}$ measured from $[\mathrm{S} \mathrm{II}]$ as described in the text.

$n_{e} \simeq 100 \mathrm{~cm}^{-3}$, increasing to $800 \mathrm{~cm}^{-3}$ at the peak of the $[\mathrm{S} \mathrm{II}]$ emission, and then decreasing to a minimum of $200 \mathrm{~cm}^{-3}$.

There is a bright knot in the [S II] images at the location of the peak density. Excluding this point, the density at the IF is likely to be $560 \mathrm{~cm}^{-3}$. Using this density, assuming a central ionizing source, and that the $13.6 \mathrm{pc}(58 \mathrm{arcsec})$ projected distance from R136 is the true three-dimensional distance and that the $1.18 \mathrm{pc}$ projected thickness of the $H^{+}$zone is the true thickness, Equation (6) requires $Q_{0}=5.9 \times 10^{51} \mathrm{~s}^{-1}$. In addition to the uncertainties in projected versus true distances, this value neglects the effect the observed gradient will have in lowering the measured average $n_{e}$.

The edge-on front IF2 forms the inner edge of one of the most prominent bright arcs, located at a projected distance of $17 \mathrm{pc}(70 \mathrm{arcsec}) \mathrm{NE}$ of R136. The thickness of the $\mathrm{H}^{+}$ layer perpendicular to the direction to R136 is approximately $0.94 \mathrm{pc}$. The density at the IF along a ray from R136 into IF2 is $n_{e}=280 \pm 50 \mathrm{~cm}^{-3}$. Applying Equation (6) with the same assumption used with IF1, we find $Q_{0}=2.0 \times 10^{51} \mathrm{~s}^{-1}$.

IF4 is a bright rim lying much further out in the nebula, at a projected distance $53 \mathrm{pc}(220 \mathrm{arcsec})$ to the east of R136 on the far side of the large faint low-density region that is easily seen in Figures 7-12. This large wall is remarkably homogeneous in density and has a thickness between 3.5 and 4 arcsec all along its projected length of $33 \mathrm{pc}(140 \mathrm{arcsec})$. The limb shows up as a density enhancement, with $n_{e}=125 \pm 30 \mathrm{~cm}^{-3}$. It has a nearly constant projected distance from R136. Using the previous technique we find $Q_{0}=4.6 \times 10^{51} \mathrm{~s}^{-1}$.

The above estimates of $Q_{0}$ are all in reasonable agreement with each other and also with $Q_{0}=4.2 \times 10^{51} \mathrm{~s}^{-1}$ estimated by Crowther \& Dessart (1998) from adding up the contributions expected from the individual stars in the cluster. These results support the conclusion that the central cluster of stars dominates the ionizing radiation field throughout most of the volume of 30 Dor, and that isolated stars provide a negligible contribution out to $100 \mathrm{pc}$.

However, there is at least one region, to the SW of R136, where the observed structure indicates ionization by a different source. The IFs designated $1 *$ through $5 *$ in Figure 14 show $\mathrm{PAH}$ emission closer to R136 than the [S II] emission. The expected stratification of $\mathrm{PAH}$ and $\mathrm{H}^{+}$emission is consistent with photo-ionization by stars in the region of the $\mathrm{OB}$ association LH 99 region. These IFs form an elongated shell structure with a diameter of $15 \mathrm{pc}$ around that cluster. If this interpretation is correct, the emission-line spectrum produced from a different ionizing spectral energy distribution would be different from the bulk of the emission from 30 Dor. Unfortunately, our spectroscopic survey does not cover this particular region, so we cannot make this test.

Interestingly there are examples of regions that appear in the optical data sets as IFs which have no associated PAH emission. One such example is a shell-like structure located at R.A. $=05^{\mathrm{h}} 39^{\mathrm{m}} 4.0$, decl. $=-69^{\circ} 06^{\prime} 07^{\prime \prime} .0(\mathrm{~J} 2000)(x=326.0$, $y=60.0$ ). It has been labeled in Figure 14 as the No PAH Shell. An offset dashed line indicates the location. This large feature is difficult to detect in $\mathrm{H} \alpha$ images, but is present in the $[\mathrm{S} \mathrm{II}] / \mathrm{H} \alpha$ or $[\mathrm{S} \mathrm{II}] /[\mathrm{O} \mathrm{III}]$ ratio images as a prominent bubblelike structure north of IF 13. However there is no associated feature seen in the $8 \mu \mathrm{m}$ Spitzer images at this location. This cannot be due to obscuration, which would affect the optical data more severely. Unfortunately this region is just beyond the boundary of our spectrophotometric survey and we can draw no further conclusions about it.

\subsection{Overall Symmetry of the Ionization Structure}

The various surface brightness and ionization maps shown in Figures 7-12 all have roughly circular symmetry. The center in each case is at a point offset about 1 arcmin to the E of R136. While at first sight this might seem counter to the idea that R136 marks the center of the source of ionizing radiation, the offset is most likely an artifact of the asymmetrical distribution of gas around R136. The extensive bubble of hot X-ray-emitting gas extending off in this direction appears to be a "blow-out" like the ones observed on a smaller scale in M17 (Townsley et al. 2003; Pellegrini et al. 2007) and elsewhere. We conclude that the asymmetrical density distribution has just modestly shifted the apparent center of the ionization structure that otherwise would be centered on R136.

\subsection{Dor at a Cosmological Distance}

In Paper II, we will compare the chemical abundances obtained from a point-by-point analysis using our new data set, to the properties that would be derived if 30 Dor were viewed at a cosmological distance and its integrated light analyzed using the "strong-line" analysis techniques (Pagel et al. 1979; Kewley \& Dopita 2002; Denicoló et al. 2002) generally used in such circumstances. 


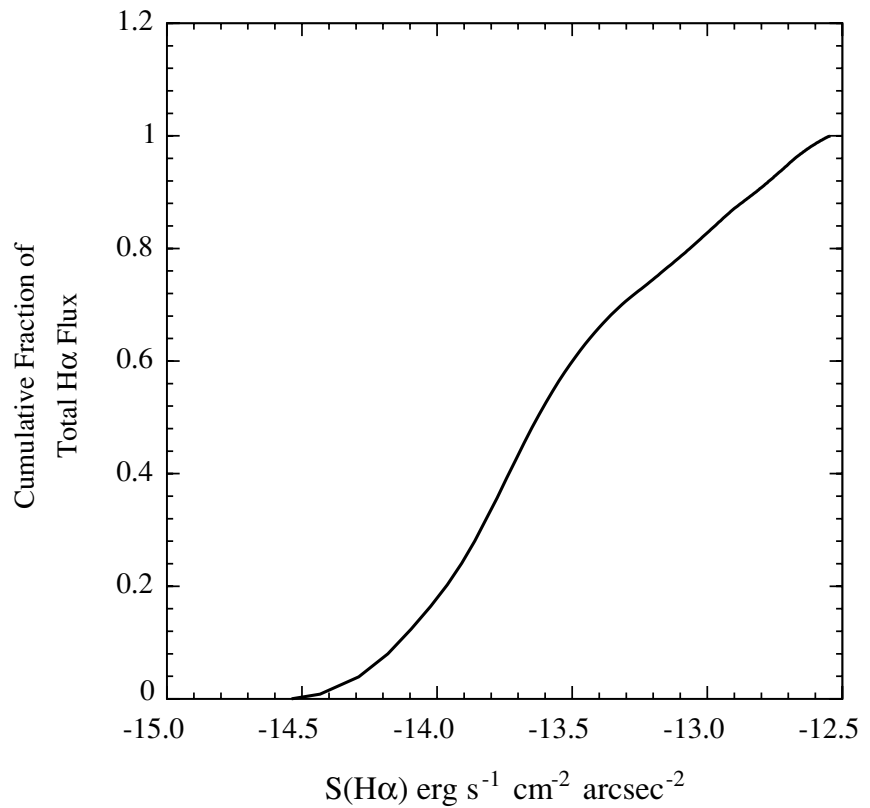

Figure 16. Cumulative histogram of the fraction of total $\mathrm{H} \alpha$ flux from pixels with $\leqslant S(H \alpha)$ from our sky- and continuum-subtracted $\mathrm{H} \alpha$ image over a $12 \times$ 13 arcmin field of view centered on R136.

It is important to not be misled by the measured properties of the prominent bright arcs that have given 30 Dor the name "Tarantula Nebula." The majority of the line emission originates in the extended regions of low surface brightness. This is demonstrated in Figure 16, a histogram of the fraction of nebular flux from regions with a surface brightness $\leqslant S(\mathrm{H} \alpha)$ for our $12 \times$ $13 \mathrm{arcmin}^{2}$ sky—and continuum-subtracted SOAR H $\alpha$ image. The bright arcs have a characteristic $\mathrm{H} \alpha$ surface brightness $S(\mathrm{H} \alpha) \geqslant 2 \times 10^{-13} \mathrm{erg} \mathrm{cm}^{-2} \mathrm{~s}^{-1} \operatorname{arcsec}^{-2}$, while $50 \%$ of the integrated $\mathrm{H} \alpha$ emission over our field originates from regions with a surface brightness an order of magnitude fainter than the bright arcs.

\section{CONCLUSIONS}

We have obtained and are making publicly available a dense grid of long-slit optical spectra which measure key emission lines in the $\lambda \lambda 4100-7400$ wavelength region over a $140 \times 80 \mathrm{pc}^{2}$ $\left(10 \times 6 \operatorname{arcmin}^{2}\right)$ region of 30 Dor. These spectra were taken at 37 slit positions and then extracted and measured as 4238 individual one-dimensional spectra. The resulting "data cube" of emission-line intensity measurements is also publicly available. Supplementary spectra at a few additional slit positions extended the coverage to a wider wavelength range.

We also obtained a set of subarcsecond-resolution direct images in multiple narrowband filters, covering a $170 \times 180 \mathrm{pc}^{2}$ $\left(12 \times 13 \operatorname{arcmin}^{2}\right)$ field of view, which we have used to identify and catalog a large number of structures of special interest. These include regions likely to be locally ionized by embedded stars, as well as edge-on IFs seen in both the visible and infrared and "elephant trunk" pillars. With JWST and ALMA on the horizon and the newly upgraded HST, these wellresolved structures in this nearby object will provide important calibrations for constraining the physical conditions in the PDRs of much more distant, unresolved low-metallicity extragalactic $\mathrm{H}$ II regions.

We find that the cluster of O stars centered on R136 is the dominant source of ionization for almost the entire nebula. Our measurements of the [O III] temperature does not reveal any shock-heated low-ionization gas. The observed emission-line intensity ratios indicate that photoionization is the predominant ionization mechanism. The general radial symmetry of these intensity ratios around a point on the sky near R136 (Figures 9-12) supports the idea that the central cluster is the major source of this photoionization.

Further evidence includes the absence of strong competition from other potential sources of ionizing radiation within $15 \mathrm{pc}$ of R136 (Section 5.1), the large numbers of elephant trunks pointing back toward R136 from points all over 30 Dor (Section 5.2), and the variation in the measured thickness of edge-on IFs as a function of distance from R136 (Section 5.3). In a follow-up paper, we will use these results as the basis for detailed models of the structure of 30 Doradus.

We also showed that the integrated emission-line spectrum of 30 Dor is dominated by the extensive regions of low surface brightness. The bright arcs near R136 catch the eye when one looks at direct images of 30 Dor, but are not what would actually be measured spectroscopically if 30 Dor were so far away that it could not be spatially resolved.

E.W.P. and J.A.B. gratefully acknowledge support from NSF grant AST-0305833 and NASA grants HST AR-10932 and NNX10AD05G. J.A.B. gratefully acknowledges additional support from NASA ADP grant NNX10AD05G. E.W.P. gratefully acknowledges support from NASA grant 07-ATFP07-0124 and partial support from NSF grant AST-0806147. G.J.F. gratefully acknowledges support from NASA grant 07-ATFP07-0124 and from NSF through 0607028 and 0908877.

\section{REFERENCES}

Bosch, G., Selman, F., Melnick, J., \& Terlevich, R. 2001, A\&A, 380, 137 Bosch, G., Terlevich, R., Melnick, J., \& Selman, F. 1999, A\&AS, 137, 21 Breysacher, J. 1981, A\&AS, 43, 203

Cardelli, J. A., Clayton, G. C., \& Mathis, J. S. 1989, ApJ, 345, 245

Chu, Y.-H., \& Kennicutt, R. C., Jr. 1994, ApJ, 425, 720

Clemens, J. C., Crain, J. A., \& Anderson, R. 2004, Proc. SPIE, 5492, 331

Crowther, P. A., \& Dessart, L. 1998, MNRAS, 296, 622

Denicoló, G., Terlevich, R., \& Terlevich, E. 2002, MNRAS, 330, 69

Elliott, K. H., Goudis, C., Meaburn, J., \& Tebbutt, N. J. 1977, A\&A, 55, 187

Elmegreen, B. G., \& Lada, C. J. 1977, ApJ, 214, 725

Hamuy, M., Suntzeff, N. B., Heathcote, S. R., Walker, A. R., Gigoux, P., \& Phillips, M. M. 1994, PASP, 106, 566

Hester, J. J., et al. 1996, AJ, 111, 2349

Hunt, L. K., \& Hirashita, H. 2009, A\&A, 507, 1327

Indebetouw, R., et al. 2009, ApJ, 694, 84 (I09)

Kewley, L. J., \& Dopita, M. A. 2002, ApJS, 142, 35

Krabbe, A. C., \& Copetti, M. V. F. 2002, A\&A, 387, 295

Macri, L. M., Stanek, K. Z., Bersier, D., Greenhill, L. J., \& Reid, M. J. 2006, ApJ, 652,1133

Mathis, J. S., Chu, Y.-H., \& Peterson, D. E. 1985, ApJ, 292, 155

Meaburn, J. 1984, MNRAS, 211, 521

Meixner, M., et al. 2006, AJ, 132, 2268

Osterbrock, D. E., \& Ferland, G. J. 2006, in Astrophysics of Gaseous Nebulae and Active Galactic nuclei, ed. D. E. Osterbrock \& G. J. Ferland (2nd. ed.; Sausalito, CA: Univ. Science Books)

Oey, M. S., Parker, J. S., Mikles, V. J., \& Zhang, X. 2003, AJ, 126, 2317

Oey, M. S., Watson, A. M., Kern, K., \& Walth, G. L. 2005, AJ, 129, 393

Pagel, B. E. J., Edmunds, M. G., Blackwell, D. E., Chun, M. S., \& Smith, G. 1979, MNRAS, 189, 95

Parker, J. W. 1993, AJ, 106, 560

Parker, J. W., \& Garmany, C. D. 1993, AJ, 106, 1471

Peimbert, A. 2003, ApJ, 584, 735

Pellegrini, E. W., Baldwin, J. A., Ferland, G. J., Shaw, G., \& Heathcote, S. 2009, ApJ, 693, 285

Pellegrini, E. W., et al. 2007, ApJ, 658, 1119

Poglitsch, A., Krabbe, A., Madden, S. C., Nikola, T., Geis, N., Johansson, L. E. B., Stacey, G. J., \& Sternberg, A. 1995, ApJ, 454, 293 
Robert, C., Pellerin, A., Aloisi, A., Leitherer, C., Hoopes, C., \& Heckman, T. M. 2003, ApJS, 144, 21

Schaller, G., Schaerer, D., Meynet, G., \& Maeder, A. 1992, A\&AS, 96, 269

Schnurr, O., Moffat, A. F. J., Villar-Sbaffi, A., St-Louis, N., \& Morrell, N. I. 2009, MNRAS, 395, 823

Scowen, P. A., et al. 1998, AJ, 116, 163

Selman, F., Melnick, J., Bosch, G., \& Terlevich, R. 1999, A\&A, 341, 98

Smith, R. C., \& MCELS Team. 1998, PASA, 15, 163
Townsley, L. K., Broos, P. S., Feigelson, E. D., Brandl, B. R., Chu, Y.-H. Garmire, G. P., \& Pavlov, G. G. 2006, AJ, 131, 2140

Townsley, L. K., Feigelson, E. D., Montmerle, T., Broos, P. S., Chu, Y.-H., \& Garmire, G. P. 2003, ApJ, 593, 874

Tsamis, Y. G., Barlow, M. J., Liu, X.-W., Danziger, I. J., \& Storey, P. J. 2003, MNRAS, 345, 186

Walborn, N. R., \& Blades, J. C. 1997, ApJS, 112, 457

Walborn, N. R., Maíz-Apellániz, J., \& Barbá, R. H. 2002, AJ, 124, 1601

Zavagno, A., et al. 2010, A\&A, 518, L101 